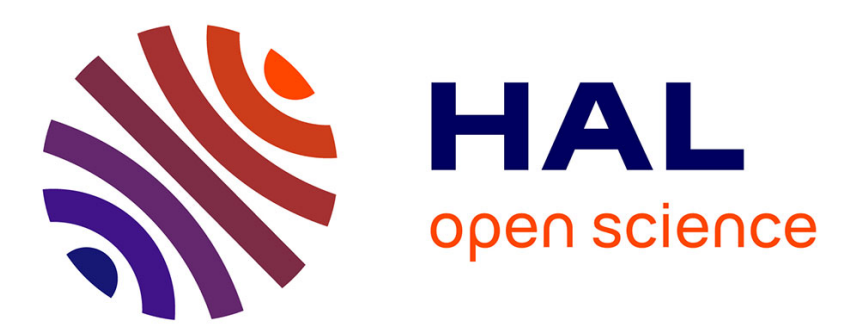

\title{
Topological characterization of toroidal chaos: A branched manifold for the Deng toroidal attractor
}

Sylvain Mangiarotti, Christophe Letellier

\section{To cite this version:}

Sylvain Mangiarotti, Christophe Letellier. Topological characterization of toroidal chaos: A branched manifold for the Deng toroidal attractor. Chaos: An Interdisciplinary Journal of Nonlinear Science, 2021, 10.1063/5.0025924. hal-03110714

\section{HAL Id: hal-03110714 \\ https://hal.science/hal-03110714}

Submitted on 14 Jan 2021

HAL is a multi-disciplinary open access archive for the deposit and dissemination of scientific research documents, whether they are published or not. The documents may come from teaching and research institutions in France or abroad, or from public or private research centers.
L'archive ouverte pluridisciplinaire HAL, est destinée au dépôt et à la diffusion de documents scientifiques de niveau recherche, publiés ou non, émanant des établissements d'enseignement et de recherche français ou étrangers, des laboratoires publics ou privés. 


\title{
Topological characterization of toroidal chaos: a branched manifold for the Deng toroidal attractor
}

\author{
Sylvain Mangiarotti ${ }^{1, \text { a) }}$ and Christophe Letellier $^{2, \text { b) }}$ \\ 1) Centre d'Études Spatiales de la Biosphère, UPS-CNRS-CNES-IRD-INRA, \\ Observatoire Midi-Pyrénées, 18 avenue Édouard Belin, 31401 Toulouse, \\ France \\ ${ }^{2)}$ Rouen Normandie University - CORIA, Campus Universitaire du Madrillet, \\ F-76800 Saint-Etienne du Rouvray, France
}

(Dated: 13 December 2020)

When a chaotic attractor is produced by a three-dimensional strongly dissipative system, its ultimate characterization is reached when a branched manifold - a template - can be used to describe the relative organization of the unstable periodic orbits around which it is structured. If topological characterization was completed for many chaotic attractors, the case of toroidal chaos - a chaotic regime based on a toroidal structure - is still challenging. We here investigate the topology of toroidal chaos, first by using an inductive approach, starting from the branched manifold for the Rössler attractor. The driven van der Pol system — in Robert Shaw's form - is used as a realization of that branched manifold. Then, using a deductive approach, the branched manifold for the chaotic attractor produced by the Deng toroidal system is extracted from data.

Dedicated to the 80th birthday of Otto E. Rössler

Chaos is related to the unpredictability of natural behaviors like atmospheric conditions since Edward N. Lorenz investigated a simple model for the Rayleigh-Bénard convection. He also described the architecture of the so-called Lorenz attractor in terms of "isopleth", showing that the trajectory was within a surface. A few years later, William introduced the concept of branched manifold to characterize this surface. With Joan Birman, he later linked branched manifold with the knot theory. Otto E. Rössler converted it into his intuitive "paper model" to describe the architecture of chaos. Rössler then constructed a zoology of chaos by proposing topologically inequivalent attractors. He was also looking for chaotic behaviors structured around torus, now called toroidal chaos. Nevertheless, no branched manifold was proposed to characterize it. Typically, a branched manifold is made of strips which require welldefined edges. Since, by definition, a torus is a surface without boundary, constructing a branched manifold for toroidal chaos is a challenging problem. This is here addressed by inserting an "allowed slit" as Rössler introduced in his "blender" for describing the so-called Rössler attractor.

\footnotetext{
a) Electronic mail: sylvain.mangiarotti@ird.fr

b) http://www.atomosyd.net/spip.php?article1; Electronic mail: christophe.letellier@coria.fr
}

\section{INTRODUCTION}

Since the seminal paper by Lorenz on the sensitivity to initial conditions presented by some solutions to simple dynamical systems, ${ }^{1}$ chaotic attractors are described by using a topological approach. The great difference with a geometrical approach is that in topology, distance is not important: what counts is the relative organization. This branch of mathematics was initiated by Leibniz. ${ }^{2}$ The first paper about the analysis situs was published in 1771 by Alexandre-Théophile Vandermonde and is devoted to the moves of knight on a chessboard. ${ }^{3}$ Then Gauss introduced one of the relevant topological invariant which is still used in the description of chaotic attractors, namely the linking number. ${ }^{4,5}$ Knot theory was initiated in 1848 by Johann Listing, ${ }^{6}$ and became intensively investigated in the late 19th century, as well exemplified by Peter Gunthrie Tait. ${ }^{7-9}$ A breakthrough was then introduced by Henri Poincaré under the name of analysis situs. ${ }^{10}$ His objective was to investigate manifold with topological invariants. He also developed a qualitative approach of the solution to nonlinear differential equations. ${ }^{11,12}$ In the dynamical systems theory, solutions to strongly dissipative systems are sketched by surface which can be split and joined, leading to the concept of branched manifold as introduced by Williams. ${ }^{13}$ With his intuitive approach, Otto E. Rössler designated branched manifold as "paper sheet model" and used them to describe the Rössler attractor as the union of a "normal" strip (without half-twist) and a Möbius strip (with one half-twist). ${ }^{14}$ Rössler was used to distinguish different chaotic attractors by sketching them with paper model. ${ }^{14-16}$ 
The population of unstable periodic orbits of the Lorenz system was extensively investigated by Joan Birman and Robert F. Williams. ${ }^{17,18}$ Branched manifold was considered as a knotholder since periodic orbits are knots. To explain experimental data with the concept of branched manifold, Robert Gilmore and co-workers used linking numbers. ${ }^{19}$ He termed "template" the obtained branched manifold. Many chaotic attractors produced by three-dimensional strongly dissipative systems were then characterized by branched manifolds. ${ }^{20-32}$ Nevertheless, the case of toroidal chaos was left as an open problem. ${ }^{33}$ The first reason is that, to the best of our knowledge, there are only a few toroidal chaotic attractors produced by three-dimensional dissipative systems, namely by the Langford system, ${ }^{34}$ the Deng toroidal system, ${ }^{35}$ and, the Li system. ${ }^{36}$ There is a system proposed by Rössler but it only produces a metastable toroidal chaos. ${ }^{37,38}$ The second reason is that toroidal surface has no boundary, a property required for constructing branched manifold. ${ }^{39}$ It is therefore rather difficult to determine a partition of the attractor and to construct a symbolic dynamics, two steps which greatly facilitates the topological characterization. ${ }^{27}$

We therefore here investigate the challenging problem of providing a topological characterization of toroidal chaos. The subsequent part of this paper is organized as follows. Section II describes, using an inductive approach, the construction of a branched manifold for toroidal chaos from the template associated with the Rössler attractor. Section III provides an example of toroidal chaos described by the branched manifold so obtained: this is the case of the driven van der Pol system. Section IV discusses, according to a deductive approach, the identification of the branched manifold for the toroidal chaotic attractor produced by the Deng toroidal system. Section V gives some conclusions to this work and suggests some perspectives.

\section{THE SIMPLEST BRANCHED MANIFOLD FOR TOROIDAL CHAOS}

In topology, a ring torus is homeomorphic to the Cartesian product of two circles $\mathrm{S}^{1} \times \mathrm{S}^{1}$. It is a compact two-manifold of genus- 1 which can be described as a quotient of the Cartesian plane under the identification

$$
(x, y) \sim(x+1, y) \sim(x, y+1) .
$$

This can be sketched as in Fig. 1. This procedure will be used for constructing a branched manifold for toroidal chaos.

As shown by Curry and Yorke, one possible scenario for getting toroidal chaos is to introduce a folding on the torus. ${ }^{40}$ This can be obtained by

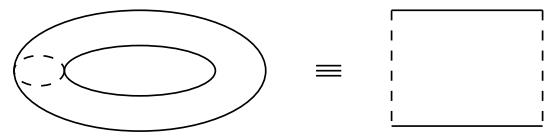

FIG. 1. A genus-1 torus can be constructed from a rectangle whose edges are joined (the top with the bottom, the left with the right).

pinching the torus, stretching it, folding it, and squeezing the surface to retrieve a simple tube (Fig. 2). Note that to get mixing properties, it is required to cut and glue the toroidal surface to produce a simply folded torus: otherwise it is only pleated and there is no mixing, that is, it can only lead to quasiperiodic regime. From Fig. 2(d), it can be seen that the folding produces three layers superimposed: it is therefore necessary to use three strips to describe a folded torus.

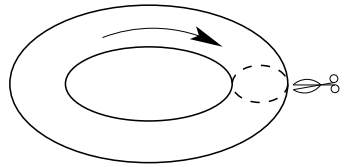

(a) Cutting

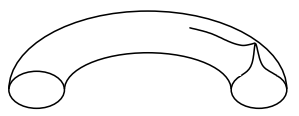

(c) Pinching \& stretching

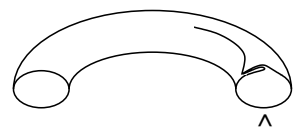

(e) Squeezing

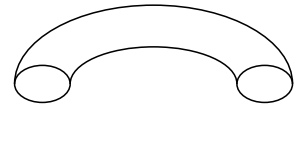

(b) Opening

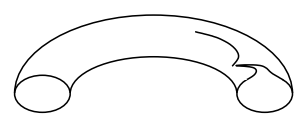

(d) Folding

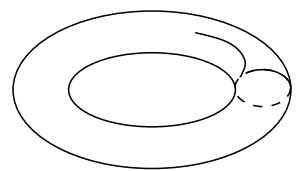

(f) Gluing
FIG. 2. Construction of a folded torus starting from a regular one (a). First, cut it, open it (b) and pinch it (c). Once stretched, fold it (d), and then squeeze it (d). Glue it back (f).

The simplest example of a chaotic attractor with a single folding mechanism is the (nontoroidal) Rössler attractor which is described by the branched manifold depicted in Fig. $3{ }^{14,27}$ To respect the convention of drawing attractor with a clockwise flow, ${ }^{41,42}$ we observe the Rössler attractor from the bottom [Fig. 3(a)]. The branched manifold can be drawn as a "paper model" [Fig. $3(\mathrm{a})]$ as used by Rössler. ${ }^{14-16}$ It can also be sketched for a more explicit representation by introducing an "allowed slit" between the two strips [Fig. 3(b)]. It is thus clearly seen that there is a "normal" strip (labelled "0" since with no halftwist) and a Möbius strip (labelled "1" since with one half-twist).

We now describe the left part of the branched manifold from the top to the bottom [Fig. 3(b)]. It is made of a splitting chart to introduce edges between strip " 0 " and strip " 1 ". Then, the local 


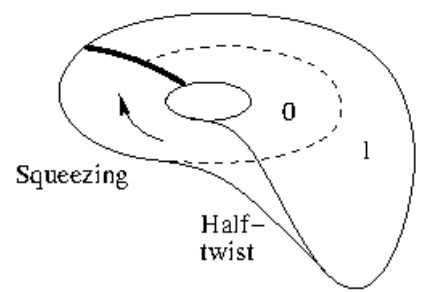

(a) Paper sheet model

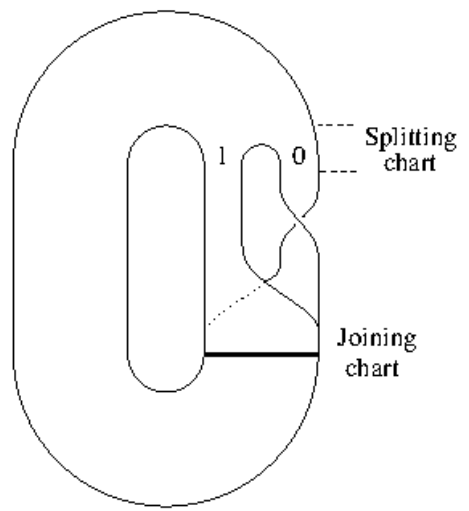

(b) Direct branched manifold

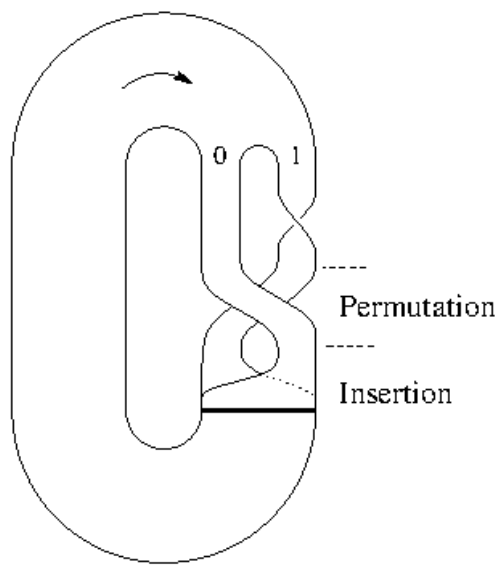

(c) Standard branched manifold

FIG. 3. Branched manifold for the Rössler attractor in the form of a "paper sheet model" (a), drawn in a direct representation (b), and with the standard insertion convention (c).

torsions are drawn one negative (anti-clockwise) half-twist for strip " 1 " and none for strip "0". The two strips are joined into a single one at the thick horizontal line [Fig. 3(b)] where a Poincaré section would be ideally computed. It is convenient to use a standard insertion convention for joining the strips: ${ }^{22}$ from the back to the front, and from the left to the right, leading to the standard branched manifold shown in Fig. 3(c).

It is thus possible to describe this branched manifold by a single linking matrix

$$
L_{i j}=\left[\begin{array}{cc}
0 & -1 \\
-1 & -1
\end{array}\right]
$$

where the strips are ordered from the centre of the attractor to its periphery. ${ }^{42}$ We use a double bracket ] to designate the joining chart were strips are squeezed. ${ }^{42}$ A simple bar ("l") would mean that the strips are still distinguished (see the case where two linkers are combined in the next section). Elements $L_{i i}$ encode the local torsion of the $i$ th strip: $L_{00}=0$ for strip " 0 " and $L_{11}=-1$ for strip "1". Off-diagonal elements $L_{i j}=-1$ $(i \neq j)$ encode the permutation between the $i$ th and the $j$ th strips: here, strip " 1 " is permuted once with strip " 0 " in the anti-clockwise direction. From this linking matrix and the orbital sequence designating periodic orbits, there is an algorithm to compute the linking numbers which quantify the number of times one orbit circles another one ( $\operatorname{see}^{27,43,44}$ for details).

To get a branched manifold for a folded torus [Fig. 2(f)], we saw that three strips are required. The branched manifold for the Rössler attractor must be completed with a third strip. To do that, we start from the folded torus, insert an "allowed slit" as indicated by the symbol " $\wedge$ " in Fig. 2(e). We thus obtain the branched manifold drawn in Fig. 4 which is described by the linking matrix

$$
M_{i j}=\left[\begin{array}{ccc}
0 & -1 & -1 \\
-1 & -1 & -1 \\
-1 & -1 & 0
\end{array}\right] .
$$

Strips are labelled 0, 1 and 0', from the center of the attractor to its periphery. We choose 0' to indicate that it is "glued" to strip 0 when the toroidal structure is reconstructed. The common convention with symbolic dynamics is to use the natural order to label the branches, ${ }^{45}$ an order that we combine with the local torsion to respect the parity of the strip: an even (odd) integer for an order preserving (reversing) strip). The symbol 0' should be manipulated as if it was a " 2 ". The simplest toroidal chaos is therefore characterized by a three-strip branched manifold, that is, by a bimodal map (with two critical points). Note that it differs from the bimodal chaos observed in the Rössler system which is characterized by the linking matrix ${ }^{27}$

$$
M_{i j}=\left[\begin{array}{ccc}
0 & -1 & -1 \\
-1 & -1 & -2 \\
-1 & -2 & -2
\end{array}\right] .
$$

To distinguish the branched manifold for a nontoroidal chaotic attractor from the one for toroidal chaos, we propose to draw the trivial part of the template, linking the joining chart — the thick horizontal line - with the splitting charts (top of the left side of the branched manifold) as suggested in Fig. 4(b) where the toroidal structure is sketched. Note that the two branched manifolds shown in Fig. 4(a) and in Fig. 4(b) are both described by the same linking matrix: the two corresponding attractors are thus topologically equivalent. 


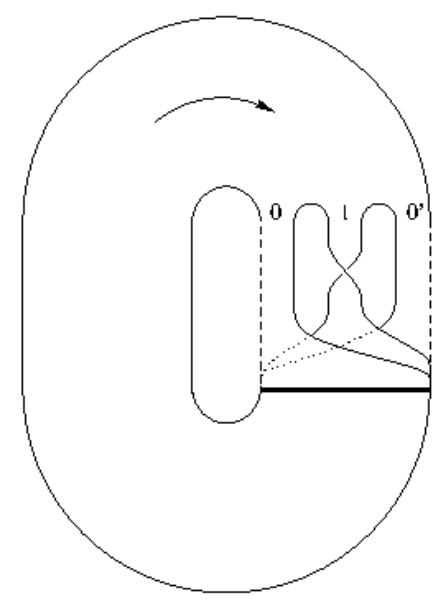

(a) Non-toroidal representation

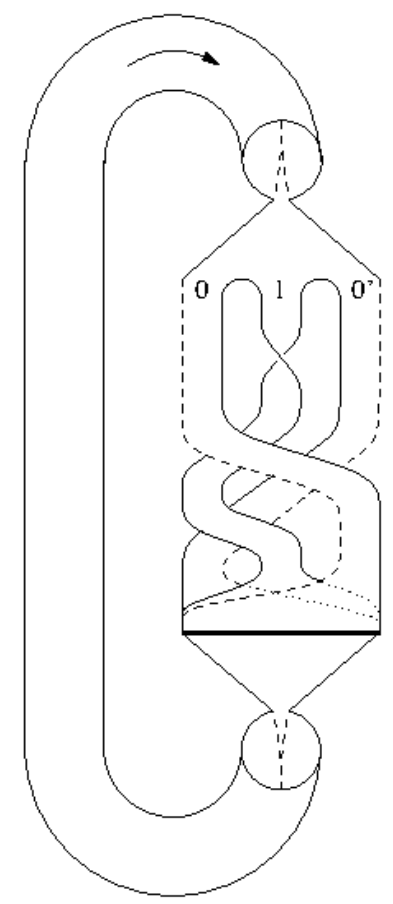

(b) Toroidal representation

FIG. 4. Branched manifold for the simply folded toroidal chaotic attractor in the classical representation (a) and in the here suggested representation (b).

Although topologically equivalent, there is a noteworthy difference between a toroidal chaotic attractor and a non-toiroidal one. Since there is two branches which are in fact the sub-branches of a single one, labelled 0 and 0 ' in our symbolic dynamics, they should not be distinguished for labelling the different periodic orbits. As a consequence, the maximum number of period- $p$ orbits in such attractor is $N_{2}(p)$ where $N_{b}(p)$ is the maximum number of period- $p$ orbits embedded within an attractor characterized by a $b$-branches firstreturn map. When the attractor is non-toroidal, the maximum number of period- $p$ orbits is $N_{3}(p)$. Since $N_{2}(p)<N_{3}(p)$, there is less periodic or- bits within the toroidal chaotic attractor associated with the branched manifold of Fig. 4(b) than in the one associated with the branched manifold drawn in Fig. 4(a).

\section{A DYNAMICAL SYSTEM FOR THE SIMPLEST TOROIDAL CHAOS}

By modifying the van der Pol system

$$
\left\{\begin{array}{l}
\dot{x}=y \\
\dot{y}=\alpha x+\left(1-\beta x^{2}\right) y
\end{array}\right.
$$

into

$$
\left\{\begin{array}{l}
\dot{x}=\alpha y+\left(1-\beta y^{2}\right) x \\
\dot{y}=-x
\end{array}\right.
$$

and applying a periodic driving term not on the acceleration but on the velocity, Robert Shaw got the driven oscillator ${ }^{46}$

$$
\left\{\begin{array}{l}
\dot{x}=\alpha y+\left(1-\beta y^{2}\right) x \\
\dot{y}=-x+u \\
\dot{u}=v \\
\dot{v}=-\omega^{2} u .
\end{array}\right.
$$

This is a semi-dissipative (or semi-conservative) system, since the dissipative van der Pol system is driven by a conservative harmonic oscillator. ${ }^{47}$ This system has thus a continuum of attractors and the initial conditions must be specified to ensure the reproductibility of the results. A toroidal chaos is obtained with Shaw's parameter values as shown in Fig. 5. Due to the conservation of energy for the harmonic oscillator, there is an integral invariant which allows to reduce by one the dimension of the state space. Moreover, we did not observe self-crossing of the attractor when embedded within our reconstructed three-dimensional space, leading to the possibility to propose a branched manifold for this toroidal attractor, indeed.
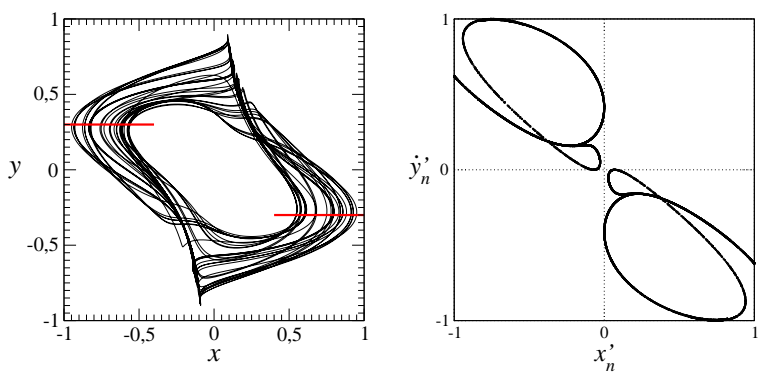

FIG. 5. Toroidal chaos produced by the driven van der Pol system (6). Parameter values: $\alpha=0.7, \beta=10$, and $\omega=\frac{\pi}{2}$. Initial conditions: $x_{0}=0.1, y_{0}=0.1$, $u_{0}=A=0.25$, and $v_{0}=0$.

This system has an inversion symmetry defined 
as

$$
\Gamma=\left[\begin{array}{cccc}
-1 & 0 & 0 & 0 \\
0 & -1 & 0 & 0 \\
0 & 0 & -1 & 0 \\
0 & 0 & 0 & -1
\end{array}\right]
$$

The system is said to be equivariant under the action of the inversion $\Gamma$, that is, it obeys to ${ }^{29}$

$$
\Gamma \cdot f(x)=f(\Gamma \cdot x) .
$$

The toroidal attractor (Fig. 5, left panel) has two foldings, one (top of the attractor) being mapped into the other (bottom of the attractor) by the inversion. In order to exhibit these two foldings, we used the Poincaré section made of the two components ${ }^{25,41,48}$

$$
\mathcal{P}_{ \pm}=\left\{\left(x_{n}^{\prime}, y_{n}^{\prime}\right) \in \mathbb{R}^{2} \mid y_{n}= \pm 0.3, \dot{y}_{n} \gtrless 0\right\}
$$

In the Poincaré section, each component is renormalized within a unit square. The inversion symmetry is obvious in this two-component Poincaré section (Fig. 5, right panel).

It is possible to modd out the symmetry by using a change of coordinates. ${ }^{49,50}$ Since the two oscillators, the modified van der Pol $(x, y)$ and the driving harmonic oscillator $(u, v)$ are rather independent, one may treat the system by blocks and use the change of coordinates

$$
\varphi=\mid \begin{aligned}
& X=x^{2}-y^{2} \\
& Y=2 x y \\
& U=u^{2}-v^{2} \\
& V=2 u v .
\end{aligned}
$$

The new governing equations are thus

$$
\mid \begin{aligned}
& \dot{X}=2 x \dot{x}-2 y \dot{y} \\
& \dot{Y}=2 \dot{x} y+2 x \dot{y} \\
& \dot{U}=2 u \dot{u}-2 v \dot{v} \\
& \dot{V}=2 \dot{u} v+2 u \dot{v} .
\end{aligned}
$$

Using that

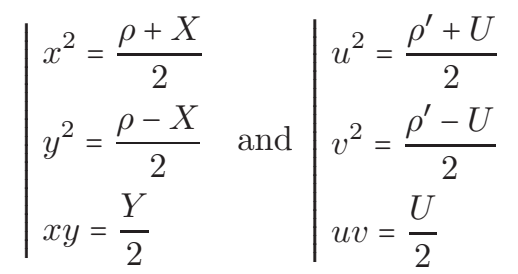

where $\rho=\sqrt{X^{2}+Y^{2}}=x^{2}+y^{2}$ and $\rho^{\prime}=\sqrt{U^{2}+V^{2}}=$ $u^{2}+v^{2}$, we get

$$
\left\{\begin{aligned}
\dot{X} & =\rho+X+(a+1) Y-\frac{b}{2} Y^{2} \\
& -\sqrt{(\rho-X)\left(\rho^{\prime}+U\right)} \\
\dot{Y}= & (a-1) \rho-(a+1) X+Y+\frac{b}{2} X Y \\
& \quad-\frac{b}{2} \rho Y+\sqrt{(\rho+X)\left(\rho^{\prime}+U\right)} \\
\dot{U}= & \left(1+\omega^{2}\right) V \\
\dot{V} & =\left(1-\omega^{2}\right) \rho^{\prime}-\left(1+\omega^{2}\right) U
\end{aligned}\right.
$$

To obtain the image of the symmetric toroidal chaos shown in Fig. 5, one may also apply the coordinate transformation $\varphi$ directly to the original attractor, leading to the toroidal chaos with no residual symmetry (Fig. 6).
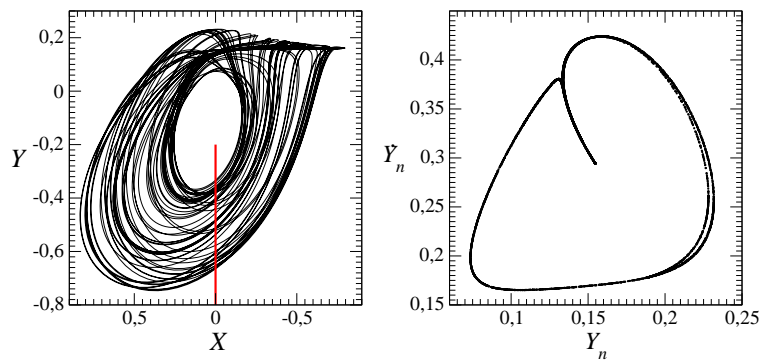

FIG. 6. Toroidal chaos produced by the image of the driven van der Pol system (6). Parameter values: $\alpha=$ $0.7, \beta=10$, and $\omega=\frac{\pi}{2}$. Initial conditions: $X_{0}=0$, $Y_{0}=0.02, U_{0}=A^{2}=0.25^{2}$, and $V_{0}=0$.

A single folding is observed in this image attractor as revealed by the Poincaré section

$$
\mathcal{P}_{\mathrm{I}}=\left\{\left(V_{n}, \dot{V}_{n}\right) \in \mathbb{R}^{2} \mid U_{n}=0, \dot{U}_{n}>0\right\}
$$

shown in Fig. 6 (right panel). There is no global torsion in this attractor and it is therefore a realization of the branched manifold proposed for the simplest toroidal chaos [Fig. 4(b)]. This is checked as follows.

From the Poincaré section $\mathcal{P}_{\mathrm{I}}$, we construct a first-return angular map based on

$$
\theta_{n}=\tan ^{-1}\left(\frac{\dot{Y}_{n}-\dot{Y}_{0}}{Y_{n}-Y_{n}}\right)
$$

where $\left(Y_{0} ; \dot{Y}_{0}\right)=(0.17 ; 0.26)$. The return map is shown in Fig. 7. This map simplifies the extraction of the unstable periodic orbits by a close-return technique. As indicated by the map which has no intersection with the first bisecting line, there is no period-1 orbit. Two period-2 orbits were found. We selected the orbits encoded by (10) and (0'1) for checking the proposed branched manifold.

The symbolic sequences are identified as follows. The only strip which can be visited by these two period-2 orbits is strip 1 which is in the middle of the first-return map, where the folding is not 


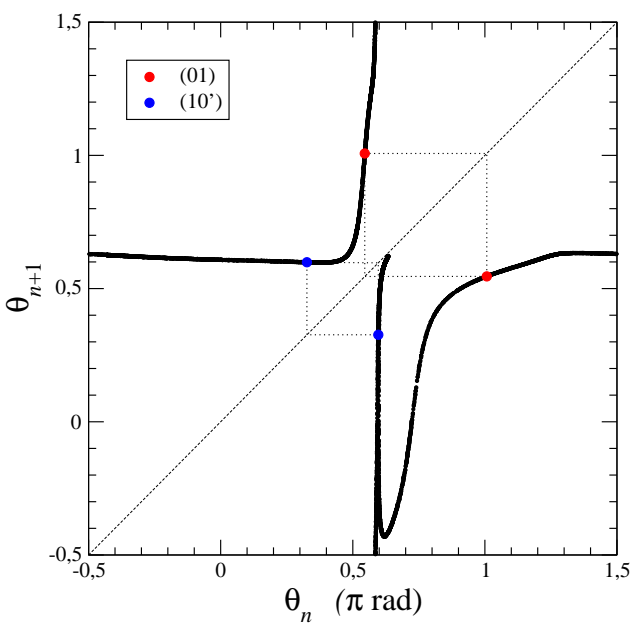

FIG. 7. First-return angular map to the Poincaré section $\mathcal{P}_{\mathrm{I}}$ of the driven van der Pol system (6). The two period-2 orbits then investigated are also plotted. Parameter values and initial conditions as in Fig. 6.

clearly unfolded (this would be rather tricky to do, if not impossible). The two period-2 orbits extracted from a chaotic trajectory are shown in Fig. 8 with the oriented crossings. Crossings between two orbits are determined in the $X-Y$ plane and signed with the third coordinate $\dot{X}$ used for constructing a three-dimensional embedding. The linking number between orbits (10) and (0'1) is equal to the half-sum of the oriented crossings, that is, to

$$
l k\left(10,0^{\prime} 1\right)=\frac{-4+2}{2}=-1 .
$$

These oriented crossings and the so-induced linking number are well-predicted by a template construction (Fig. 9).

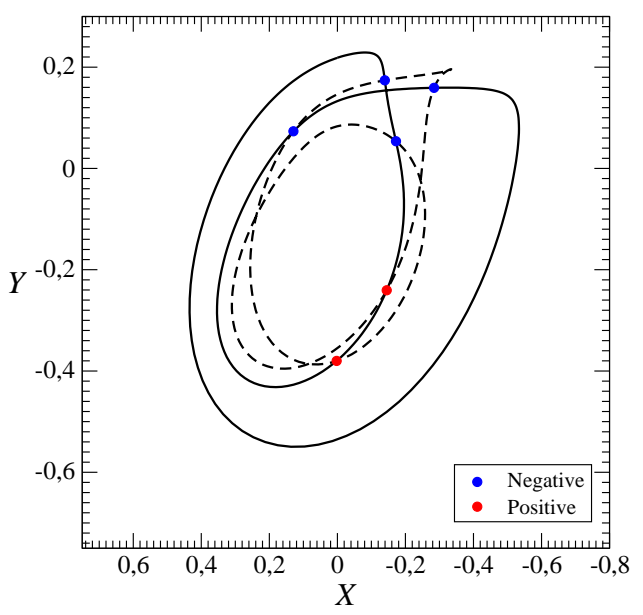

FIG. 8. Link formed from the two period-2 orbits extracted from the toroidal chaos shown in Fig. 6. $\mathrm{Pa}-$ rameter values and initial conditions as in Fig. 6.

Indeed, as in the $X-Y$ plane projection, four negative and two positive crossings are found, leading to a linking number $l k\left(10,0^{\prime} 1\right)=-1$. The image of the driven van der Pol system (6) therefore produces a toroidal chaos which is characterized by the branched manifold shown in Fig. 4(b).

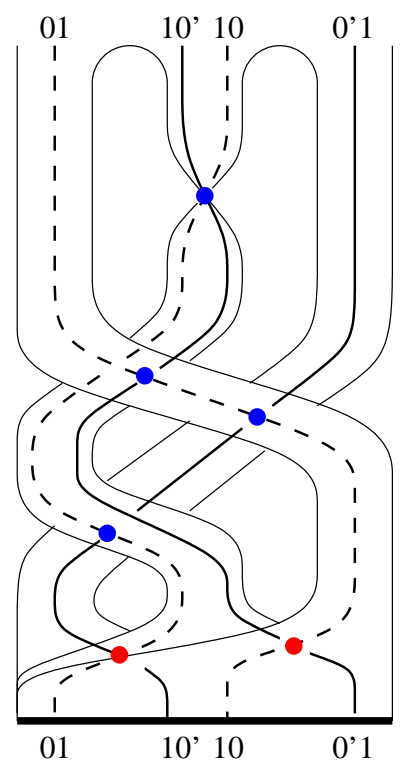

FIG. 9. Template construction of the two orbits (10) and (0'1) on the branched manifold obtained for the driven van der Pol system. Only the non trivial part of it is drawn. Same color convention for the oriented crossings as in Fig. 8.

\section{TOPOLOGICAL CHARACTERIZATION OF THE DENG TOROIDAL ATTRACTOR}

The Deng toroidal system is a set of three ordinary differential equations reading as ${ }^{35}$

$$
\left\{\begin{aligned}
\dot{x}= & (\lambda x-\mu y) \\
& +(2-z)\left[\alpha x\left(1-\frac{x^{2}+y^{2}}{R^{2}}\right)-\beta y\right] \\
\dot{y}= & z(\mu x+\lambda y) \\
& +(2-z)\left[\alpha y\left(1-\frac{x^{2}+y^{2}}{R^{2}}\right)+\beta x\right] \\
\dot{z}= & \frac{1}{\epsilon}\left[z\left((2-z)\left(a(z-2)^{2}+b\right)-d x\right)\right. \\
& \left.\times\left(z+m\left(x^{2}+y^{2}\right)-\eta\right)-\epsilon c(z-1)\right]
\end{aligned}\right.
$$

This system produces a toroidal chaotic attractor (Fig. 10).

The toroidal structure is easily exhibited by using one of the Poincaré section

$$
\mathcal{P}_{ \pm} \equiv\left\{\left(x_{n}, z_{n}\right) \in \mathbb{R}^{2} \mid y_{n}=1.5, \dot{y}_{n} \gtrless 0\right\} .
$$

The Poincaré section $\mathcal{P}_{-}$is a trivial closed curve, typical of a torus (Fig. 11, left panel). The Poincaré section $\mathcal{P}_{+}$, located at the right side of the attractor shown in Fig. 10, is also a closed curve but with a folding (Fig. 11, right panel). The route to this toroidal chaos should therefore be the route described by Curry and Yorke. ${ }^{40}$ 


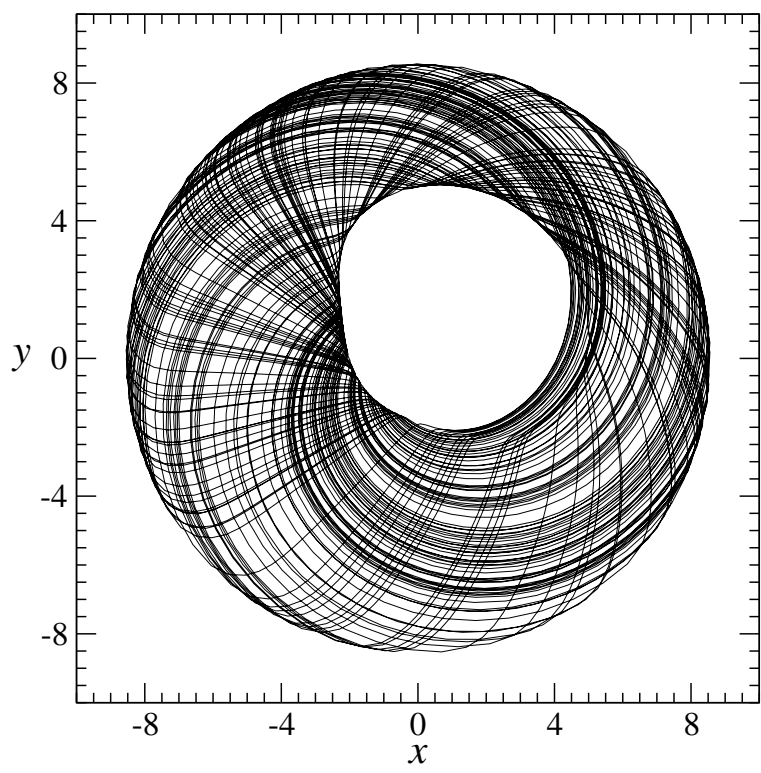

FIG. 10. Toroidal chaos produced by the Deng toroidal system (17). Parameter values: $a=3, b=0.8, c=1$, $d=0.1, m=0.05, \eta=3.312, R=10, \alpha=2.8, \beta=5$, $\epsilon=0.1, \lambda=-2$, and $\mu=1.155$.
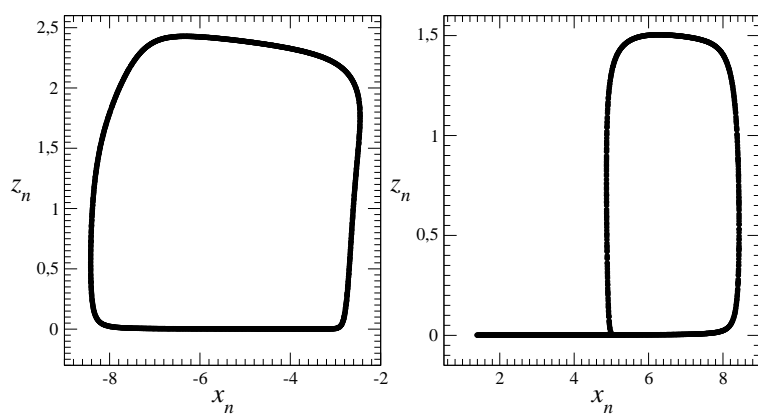

FIG. 11. Poincaré section of the toroidal chaotic attractor produced by the Deng toroidal system (17) Same parameter values as in Fig. 10.

For smaller $\mu$-values, the bifurcation diagram is quite complex, with a lot of periodic windows and period-doubling cascades [Fig. 12(a)]. For instance, there are one period-doubling cascade issued from a period-2 limit cycle $(\mu=1.18)$ and one inverse period-doubling cascade issued from a period-3 limit cycle $(\mu=1.24)$. Both lead to chaotic attractors which co-exist for $\mu \epsilon$ $[1.2107 ; 1.2120]$ within the accuracy of our bifurcation diagram, that is, according to $\mathrm{d} \mu=10^{-4}$ ) [Fig. 12(b)]. There are many bubblings. Toroidal chaos is observed for $\mu>1.058$; before the torus is not closed and, rigorously, one cannot qualify it as being toroidal chaos. An example of the two co-existing chaotic attractors is shown in Fig. 13. The two attractors can be designed as banded chaos, since they are structured according to a band which is twisted and knotted. ${ }^{51}$

We now investigate the toroidal chaos shown in Fig. 10. Since it is rather difficult to get a partition of the attractor, we use a color tracer technique as

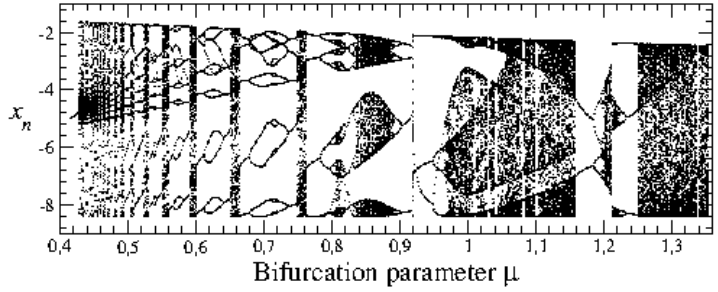

(a) Full diagram

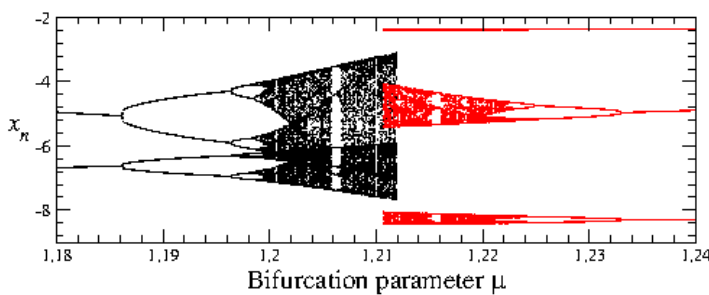

(b) Blow up

FIG. 12. Bifurcation diagram of the Deng toroidal system (17). (a) Full diagram and (b) blow up. Other parameter values as in Fig. 10.

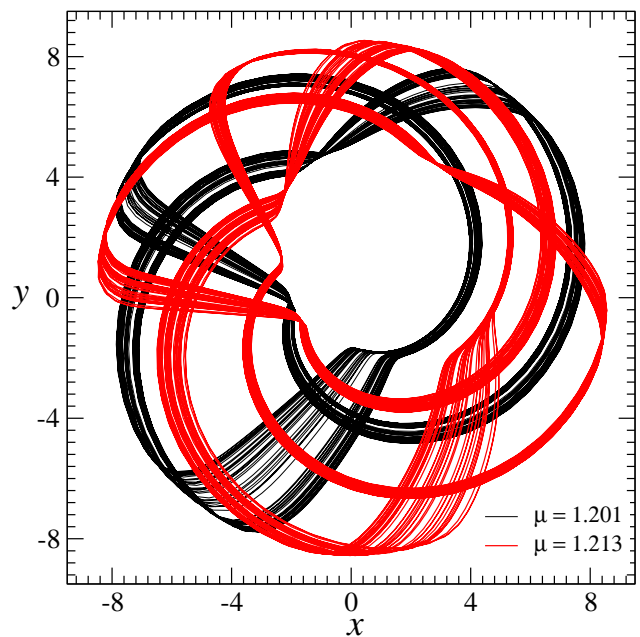

FIG. 13. Knotted banded chaos produced by the Deng toroidal system (17). Parameter values $\mu=1.211$ and others as in Fig. 10.

follows. ${ }^{52}$ Different color tracers are injected in a Poincaré section, and we followed how they are propagated up to the next intersection with the Poincaré section. Step-by-step, domains are identified. We found that a three-strip branched manifold can correctly describe the structure of this toroidal chaos [Fig. 14(a)]. It has the structure of the branched manifold we constructed by induction in Fig. 4(b) but with a global torsion of three negative half-twists (as shown in the right part of the branched manifold).

When a global torsion $\tau_{n}$ is inserted in a branched manifold, the corresponding linker is obtained by using the composition rule ${ }^{42}$

$$
\mathcal{L}^{\prime}=\mathcal{T}_{\eta}+\mathcal{L} \equiv \mid \begin{array}{ll}
T_{\eta}+L & \text { if } \eta \text { is even } \\
T_{\eta}+L^{p} & \text { if } \eta \text { is odd }
\end{array}
$$

where $\mathcal{L}^{\prime}$ corresponds to the linking matrix de- 


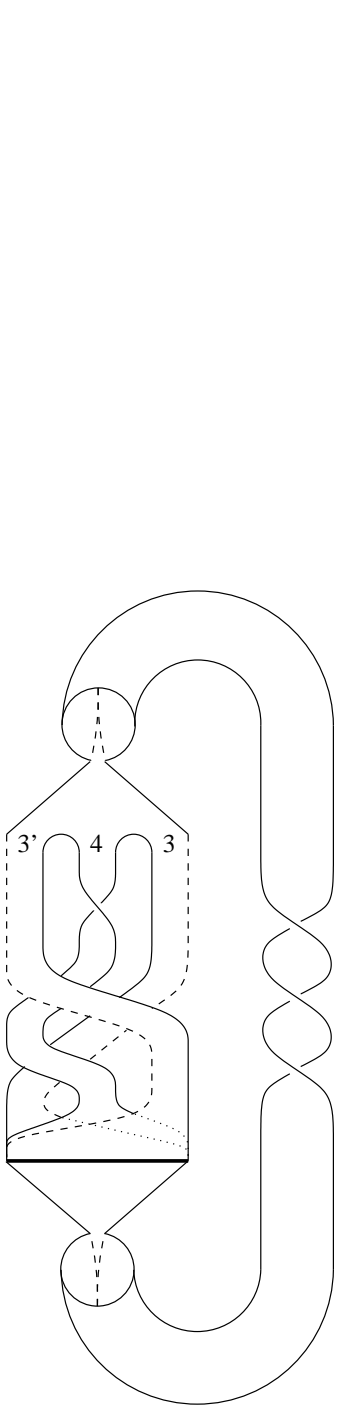

(a) Direct

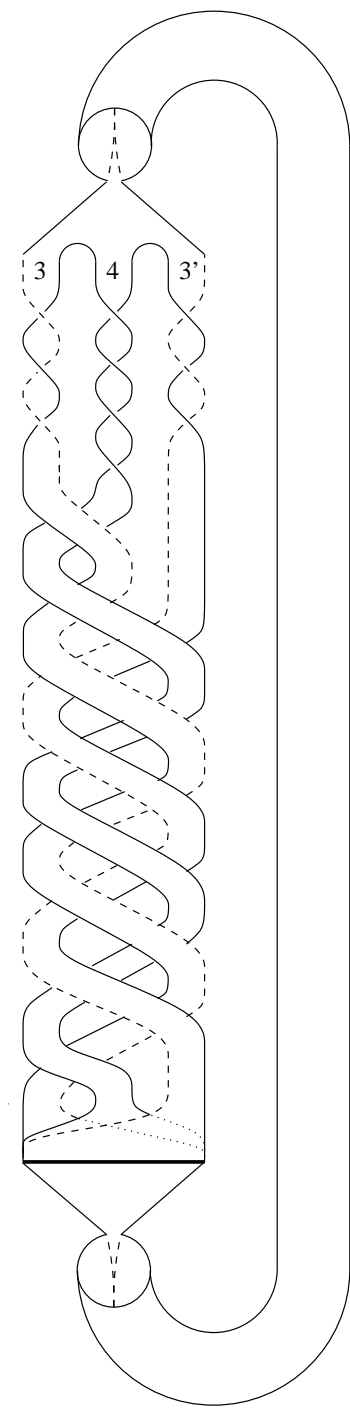

(b) Standard
FIG. 14. Branched manifold for the Deng toroidal attractor drawn in a direct representation (a), and with the standard insertion convention (b).

scribing a global $\eta$-torsion $\mathcal{T}_{\eta}$ associated with the linking matrix $T_{\eta}$ followed by a linker $\mathcal{L}$ corresponding to the linking matrix $L$. Since the global torsion is here odd, the linking matrix (2) must be permuted according to ${ }^{42}$

$$
M^{\mathrm{p}}=\left[\begin{array}{ccccc}
M_{n n} & M_{n n-1} & \ldots & M_{n 2} & M_{n 1} \\
M_{n-1 n} & M_{n-1 n-1} & \ldots & M_{n-12} & M_{n-11} \\
\vdots & \vdots & \ddots & \vdots & \vdots \\
M_{2 n} & M_{2 n-1} & \ldots & M_{22} & M_{21} \\
M_{1 n} & M_{1 n-1} & \ldots & M_{12} & M_{11}
\end{array}\right]
$$

In the present case, $M^{\mathrm{p}}=M$. Consequently, only the order of strips is permuted. The corresponding linker is thus

$$
\begin{aligned}
\mathcal{L}_{\mathrm{D}} & =\left[\begin{array}{lll}
-3 & -3 & -3 \\
-3 & -3 & -3 \\
-3 & -3 & -3
\end{array}|+| \begin{array}{ccc}
0 & -1 & -1 \\
-1 & -1 & -1 \\
-1 & -1 & 0
\end{array}\right] \\
& =\left[\begin{array}{lll}
-3 & -4 & -4 \\
-4 & -4 & -4 \\
-4 & -4 & -3
\end{array}\right]
\end{aligned}
$$

Note that the labelling of the strips was changed according to their local torsion: they are now 3,4 and 3' [Fig. 4(b)]. The natural order is obtained in using 3 ' as if it would be " 5 ".

To check the adequacy of the branched manifold described by the linker $\mathcal{L}_{\mathrm{D}}$, we proceeded as for the driven van der Pol system. We selected the Poincaré seciton $\mathcal{P}_{-}$to avoid the folding: when it is possible, it is indeed recommended to avoid the folding for constructing a first-return map. ${ }^{44}$ Then, the angle

$$
\theta_{n}=\tan ^{-1}\left(\frac{z_{n}-z_{0}}{x_{n}-x_{n}}\right)
$$

with $\left(x_{0} ; z_{0}\right)=(-0.55 ; 1.25)$ is computed for each point and the first-return map plotted as shown in Fig. 15). It does not have the overlap we were not able to remove with the driven van der Pol system. Once again there is no obvious critical point in this first-return map, meaning that the partition of the attractor is not straightforward. Since there is no intersection with the bisecting line, there is no period-1 orbit. The numerical extraction of the population of unstable periodic orbits reveals that there is no period-2 orbits.

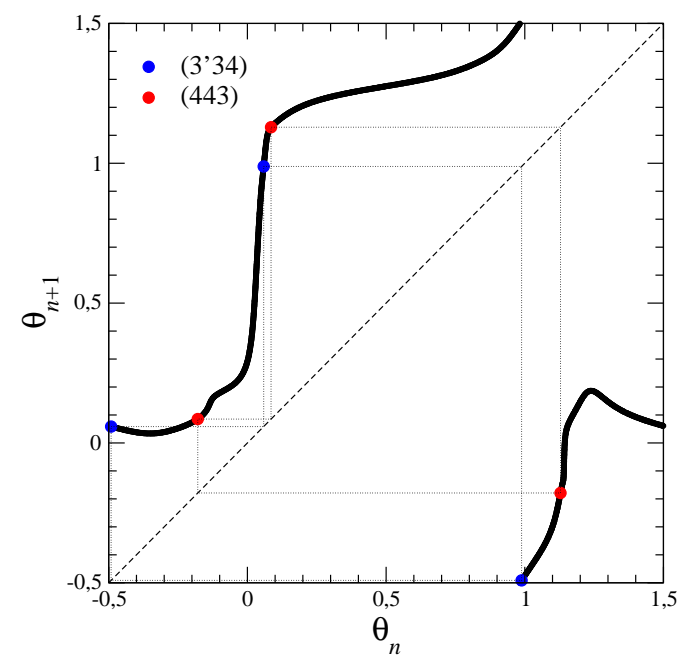

FIG. 15. Knot made of the two period-3 orbits extracted from the toroidal chaotic attractor produced by the Deng toroidal system (17). Negative crossings are plotted as blue disks. Parameter values: $\mu=1.35$ and others as in Fig. 10.

We therefore selected the two period-3 orbits (Fig. 16) to compute the linking number to compare with a template construction. After a trialand-error approach, we encode these two period-3 
orbits as (3'43) and (443), respectively. There are 30 negative crossings, leading to a linking number $l k\left(3^{\prime} 43 ; 443\right)=-15$. It is in agreement with the template construction. In fact, here, we did not use a drawing as in Fig. 9 but the algorithm for computing linking number from the linking matrix (see a brief description of its application in Appendix A). ${ }^{43}$ The branched manifold proposed in Fig. 4(b) therefore explains the toroidal chaos produced by the Deng toroidal system (17) for $\mu=1.155$.

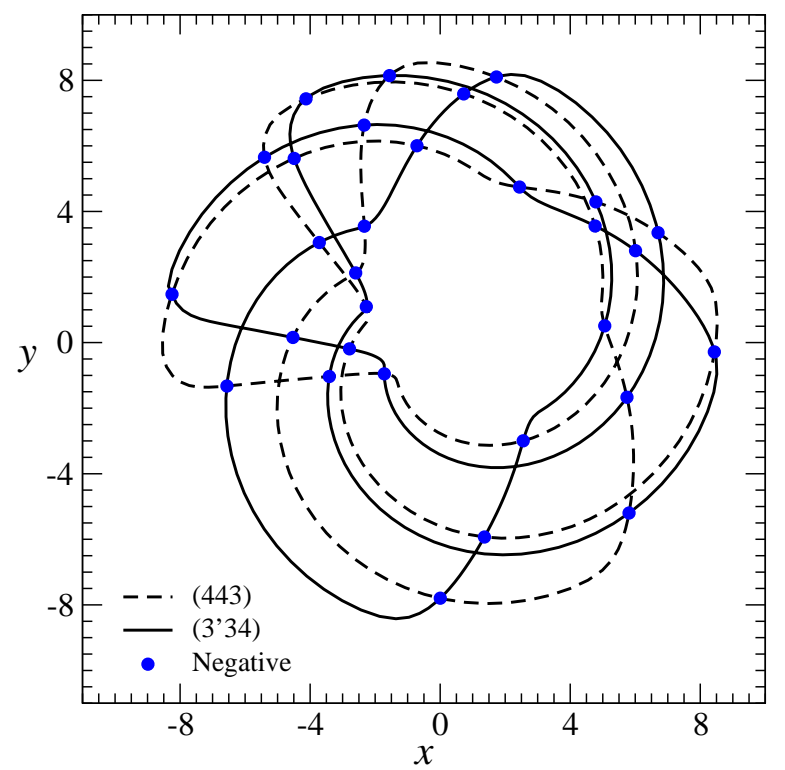

FIG. 16. Knot made of the two period-3 orbits extracted from the toroidal chaotic attractor produced by the Deng toroidal system (17). Negative crossings are plotted as blue disks. Parameter values: $\mu=1.35$ and others as in Fig. 10.

For $\mu=1.35$, that is, just before a boundary crisis which ejects the trajectory to infinity $\left(\mu_{\mathrm{BC}} \approx 1.354\right)$, and after the bubble between the period-2 and the period-3 orbits, the attractor (Fig. 17) is more developed since it is structured around more numerous unstable periodic orbits. Two period-1 orbits are found with a linking number $l k(\alpha, \beta)=-1$ where $\alpha$ and $\beta$ are the unknown orbital sequence. Such a linking number cannot be explained with a branched manifold with a negative global torsion $\tau_{3}=-3$. This attractor must be described by a branched manifold with, at least, one (most likely two) additional strips. This will be investigated in forthcoming studies.

\section{CONCLUSION}

The topology of toroidal chaos was here addressed in terms of branched manifold. The key point to open "a breach in the affordable place" was to introduce an allowed slit at distance from the folding. The largest strip described in the toroidal surface was thus split into two strips.

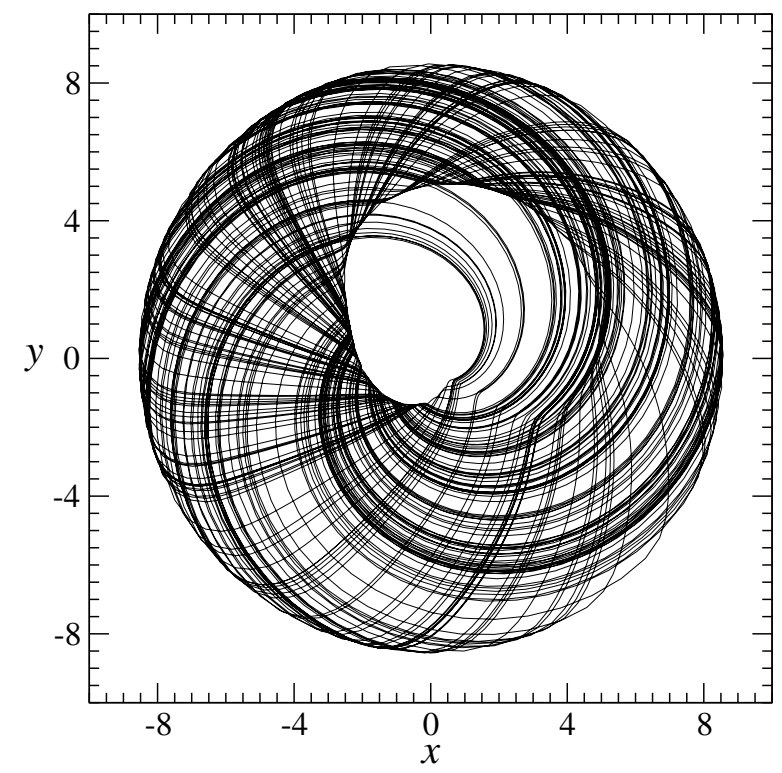

FIG. 17. Toroidal chaos produced by the Deng toroidal system (17). Parameter values: $\mu=1.35$ and others as in Fig. 10.

A simple folding was thus interpreted in terms of a three-strip branched manifold and not only with two strips as for non toroidal chaos like the Rössler attractor. We were thus able to propose a branched manifold for the image of the driven van der Pol system in Shaw's form and for the Deng toroidal system. Such a success will allow to accurately characterize toroidal chaos, at least for strongly dissipative systems, that is, when the toroidal chaos is within a thin closed surface and clearly characterized by a closed curve as a Poincaré section. This feature opens the possibility to construct a hierarchy of toroidal chaos. Thus the Deng toroidal chaos is just a chaos similar to the one produced by the driven van der Pol syste (for the form and the parameter values here investigated) but with an additional global torsion of three negative half-twists.

Further investigation will lead to construct the branched manifold for the Deng toroidal system just before the boundary crisis. This will allow to accurately understand the effect on the toroidal dynamics of the bubbling between the period-2 and period-3 limit cycles. Another system which deserves attention is the $\mathrm{Li}$ system with its rotation symmetry and its numerous foldings. Now that we unlocked the topological characterization of toroidal chaos by branched manifold, many new studies are possible for improving our understanding of toroidal chaos.

\section{DATA AVAILABILITY STATEMENT}

All the date used in this work were produced with basic codes for integrating the two systems under study by using a Runge-Kutta integration 
scheme.

${ }^{1}$ E. N. Lorenz, "Deterministic nonperiodic flow," Journal of the Atmospheric Sciences 20, 130-141 (1963).

${ }^{2}$ H. Freudenthal, "Leibniz und die Analysis Situs," Studia Leibnitiana 4, 61-69 (1972).

${ }^{3}$ A.-T. Vandermonde, "Remarques sur les problèmes de situation," Mémoires de l'Académie Royale des Sciences (Paris) , 566-574 (1771).

${ }^{4}$ C. F. Gauss, "Note dated 22 january 1833," in Werke, Vol. 5, edited by C. Schäfer (Königliche Gesellschaft der Wissenschaften zu Göttingen, Leipzig, Berlin, 1867) p. 605.

${ }^{5}$ R. L. Ricca and B. Nipoti, "Gauss' linking number revisited," Journal of Knot Theory and its Ramifications 20 , 1325-1343 (2011).

${ }^{6}$ J. B. Listing, Vorstudien zur Topologie (Vandenboeck und Ruprecht, Göttingen, 1848).

${ }^{7}$ P. G. Tait, "On knots I," Transactions of the Royal Society of Edinburgh 28, 145-190 (1877).

${ }^{8}$ P. G. Tait, "On knots II," Transactions of the Royal Society of Edinburgh 32, 327-342 (1884).

${ }^{9}$ P. G. Tait, "On knots III," Transactions of the Royal Society of Edinburgh 32, 493-506 (1884)

${ }^{10}$ H. Poincaré, "Analysis situs," Journal de l'École Polytechnique 1, 1-121 (1895).

${ }^{11} \mathrm{H}$. Poincaré, "Sur le problème des trois corps et les équations de la dynamique," Acta mathematica 13, 1270 (1890)

${ }^{12} \mathrm{H}$. Poincaré, Les méthodes nouvelles de la mécanique céleste III (Gauthier-Vilard, Paris, 1899).

${ }^{13}$ R. F. Williams, "Expanding attractors," Publications Mathématiques de l'Institut des Hautes Études Scientifiques 43, 169-203 (1974).

${ }^{14} \mathrm{O}$. E. Rössler, "Chaotic behavior in simple reaction system," Zeitschrift für Naturforschung A 31, 259-264 (1976).

${ }^{15}$ O. E. Rössler, "An equation for continuous chaos," Physics Letters A 57, 397-398 (1976).

${ }^{16}$ O. E. Rössler, "Chaos in abstract kinetics: Two prototypes," Bulletin of Mathematical Biology 39, 275-289 (1977).

${ }^{17} \mathrm{~J}$. Birman and R. F. Williams, "Knotted periodic orbits in dynamical systems I. Lorenz's equations," Topology 22, 47-82 (1983).

${ }^{18} \mathrm{~J}$. Birman and R. F. Williams, "Knotted periodic orbits in dynamical systems II. Knot holders for fibred knots," Contemporary Mathematics 20, 1-60 (1983).

${ }^{19}$ R. Gilmore, "Topological analysis of chaotic dynamical systems," Reviews of Modern Physics 70, 1455-1529 (1998).

${ }^{20}$ G. B. Mindlin, H. G. Solari, M. A. Natiello, R. Gilmore, and X.-J. Hou, "Topological analysis of chaotic time series data from the Belousov-Zhabotinskii reaction," Journal of Nonlinear Science 1, 147-173 (1991)

${ }^{21}$ G. M. Mindlin and R. Gilmore, "Topological analysis and synthesis of chaotic time series," Physica D 58, 229-242 (1992).

${ }^{22}$ N. B. Tufillaro, T. Abbott, and J. Reilly, An experimental approach to nonlinear dynamics and chaos (AddisonWesley, Redwood City, CA, 1992).

${ }^{23}$ M. Lefranc and P. Glorieux, "Topological analysis of chaotic signals from a $\mathrm{CO}_{2}$ laser with modulated losses," International Journal of Bifurcation \& Chaos 3, 643-650 (1993).

${ }^{24}$ L. Kocarev, Z. Tasev, and D. Dimovski, "Topological description of a chaotic attractor with spiral structure," Physics Letters A 190, 399-402 (1994).

${ }^{25}$ C. Letellier, P. Dutertre, and G. Gouesbet, "Characterization of the Lorenz system, taking into account the equivariance of the vector field," Physical Review E 49 3492-3495 (1994).
${ }^{26}$ T. Braun, R. R. B. Correia, and N. Altmann, "Topological model of homoclinic chaos in a glow discharge," Physical Review E 51, 4165-4168 (1995).

${ }^{27}$ C. Letellier, P. Dutertre, and B. Maheu, "Unstable periodic orbits and templates of the Rössler system: Toward a systematic topological characterization," Chaos 5, 271282 (1995).

${ }^{28}$ R. W. Ghrist and P. J. Holmes, "An ODE whose solutions contain all knots and links," International Journal of Bifurcations \& Chaos 6, 779-800 (1996).

${ }^{29}$ C. Letellier, P. Dutertre, J. Reizner, and G. Gouesbet, "Evolution of multimodal map induced by an equivariant vector field," Journal of Physics A 29, 5359-5373 (1996).

${ }^{30}$ G. Boulant, M. Lefranc, S. Bielawski, and D. Derozier, "A nonhorseshoe template in a chaotic laser model," International Journal of Bifurcation \& Chaos 08, 965-975 (1998).

${ }^{31}$ C. Letellier, E. Roulin, and O. E. Rössler, "Inequivalent topologies of chaos in simple equations," Chaos, Solitons \& Fractals 28, 337-360 (2006).

${ }^{32} \mathrm{C}$. Letellier and R. Gilmore, "The universal template is a subtemplate of the double-scroll template," Journal of Physics A 46, 065102 (2013).

${ }^{33}$ R. Gilmore, C. Letellier, and M. Lefranc, "Chaos topology," Scholarpedia 3, 4592 (2008).

${ }^{34}$ W. F. Langford, "Numerical studies of torus bifurcations," in Numerical Methods for Bifurcation Problems, International Series of Numerical Mathematics, Vol. 70, edited by T. Küpper, H. D. Mittelmann, and H. Weber (Birkhäuser, Basel, 1984) pp. 285-295.

${ }^{35}$ B. Deng, "Constructing homoclinic orbits and chaotic attractors," International Journal of Bifurcation \& Chaos 4, 823-841 (1994).

${ }^{36}$ D. Li, "A three-scroll chaotic attractor," Physics Letters A 372, 387-393 (2008).

${ }^{37}$ O. E. Rössler, "Continuous chaos: four prototype equations," Annals of the New York Academy of Sciences 316, 376-392 (1979)

${ }^{38} \mathrm{C}$. Letellier and O. E. Rössler, "Chaos: The world of nonperiodic oscillations," (Springer, Cham, Switzerland, 2020) Chap. An updated hierarchy of chaos, pp. 181-203.

${ }^{39} \mathrm{G}$. Mindlin and H. Solari, "Tori and Klein bottles in four-dimensional chaotic flows," Physica D 102, 177-186 (1997).

40 J. Curry and J. A. Yorke, "A transition from Hopf bifurcation to chaos: computer experiments with maps on $\mathbb{R}^{2}$," Lecture Notes in Mathematics 668, 48-66 (1978).

${ }^{41}$ T. D. Tsankov and R. Gilmore, "Topological aspects of the structure of chaotic attractors in $\mathbb{R}^{3}$," Physical Review E 69, 056206 (2004).

${ }^{42} \mathrm{M}$. Rosalie and C. Letellier, "Systematic template extraction from chaotic attractors: I. Genus-one attractors with an inversion symmetry," Journal of Physics A: Mathematical and Theoretical 46, 375101 (2013).

${ }^{43}$ L. Le Sceller, C. Letellier, and G. Gouesbet, "Algebraic evaluation of linking numbers of unstable periodic orbits in chaotic attractors," Physical Review E 49, 4693-4695 (1994).

${ }^{44} \mathrm{C}$. Letellier, Caractérisation topologique et reconstruction des attracteurs étranges, Ph.D. thesis, University of Paris VII, Paris, France (1994).

${ }^{45} \mathrm{H}$. Bai-Lin, Elementary symbolic dynamics and chaos in dissipative systems (World Scientific Publishing, Singapore, 1989).

${ }^{46} \mathrm{R}$. Shaw, "Strange attractor, chaotic behavior and information flow," Zeitschrift für Naturforschung A 36, 80112 (1981).

${ }^{47}$ O. Ménard, C. Letellier, J. Maquet, L. L. Sceller, and G. Gouesbet, "Analysis of a non synchronized sinusoidally driven dynamical system," International Journal of Bifurcation \& Chaos 10, 1759-1772 (2000)

${ }^{48}$ C. Letellier, , and G. Gouesbet, "Topological characterization of Reconstructed Attractors Modding Out Sym- 
metries," Journal de Physique II 6, 1615-1638 (1996).

${ }^{49}$ R. Miranda and E. Stone, "The proto-Lorenz system," Physics Letters A 178, 105-113 (1993).

${ }^{50} \mathrm{C}$. Letellier and R. Gilmore, "Covering dynamical systems: Two-fold covers," Physical Review E 63, 016206 (2001).

${ }^{51}$ E. Ott, Chaos in Dynamical Systems (Cambridge University Press, New York, 1993).

${ }^{52}$ S. Mangiarotti, M. Peyre, and M. Huc, "A chaotic model for the epidemic of Ebola virus disease in West Africa (2013-2016)," Chaos 26, 113112 (2016).

\section{Appendix A: Computing linking number}

As developed by Le Sceller and co-workers, ${ }^{43}$, there is an algorithm for computing the linking numbers from a linking matrix $L$. Let us briefly describe it for the particular case of the two period3 orbits extracted from the Deng toroidal chaos characterized by the linking matrix (20). The linking number between two orbits $\alpha$ and $\beta$ is given by

$$
l k(\alpha, \beta)=\frac{1}{2}\left[\sum_{n=1}^{p_{\alpha}} \sum_{m=1}^{p_{\beta}} L_{\alpha_{n} \beta_{m}}+N_{\mathrm{ins}}(\alpha, \beta)\right]
$$

where $\alpha_{n}\left(\beta_{m}\right)$ are the $p_{\alpha}\left(p_{\beta}\right)$ symbols used to write the orbital sequence $\alpha(\beta)$ of the period $-p_{\alpha}$ (period- $p_{\beta}$ ) orbit. $N_{\text {ins }}$ is the so-called graph of insertion constructed as follows in the explicit case of orbits (443) and (3'34).

These two orbits are made of three periodic points each, namely

$$
443 \rightarrow 434 \rightarrow 344
$$

and

$$
3^{\prime} 34 \rightarrow 343^{\prime} \rightarrow 43^{\prime} 3,
$$

respectively. These six periodic points can be ranked according to the multimodal order ${ }^{27,45}$ as

$$
343^{\prime}<344<434<443<43^{\prime} 3<3^{\prime} 34
$$

which provides the relative order of the periodic points in the Poincaré section. This is the bottom row of the graph of insertion. The top row is obtained by a permutation between the periodic point within a given order-reversing strip (labelled with an odd integer) and a permutation between the $i$ th and the $j$ th strips which are associated with an odd element $L_{i j}$ of the linking matrix. In the present case, the top row is

$$
344<343^{\prime}<434<443<43^{\prime} 3<3^{\prime} 34 .
$$

The top row is thus linked to the bottom row according to a Bernoulli shift: for instance, point 3'34 of the top row is linked to point 343' of the bottom row. Crossings between the two orbits are then counted (self-crossings are ignored). In the graph of insertion $N_{\text {ins }}\left(3^{\prime} 34,443\right)$ (Fig. 18), five crossings are counted. By definition, crossings in the graph of insertion are always positive. ${ }^{43}$ The linking number $l k\left(3^{\prime} 34,443\right)$ is thus equal to

$$
l k\left(3^{\prime} 34,443\right)=\frac{1}{2}[-3-4-12-8-8+5]=-15
$$

which is equal to the linking number counted in a plane projection of the two orbits as shown in Fig. 16.

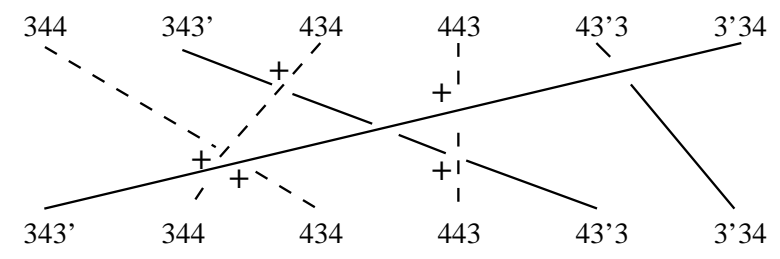

FIG. 18. Graph of insertion $N_{\text {ins }}\left(3^{\prime} 34,443\right)$ between the period-3 orbits (3'34) and (443). Five positive crossings are counted. Self-crossings are ignored. 


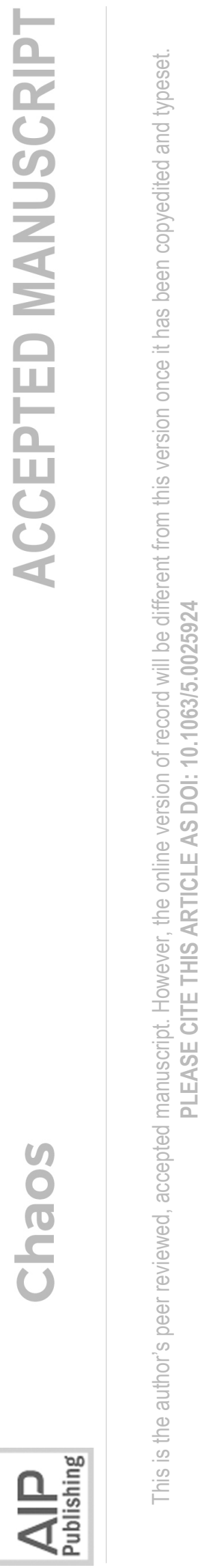




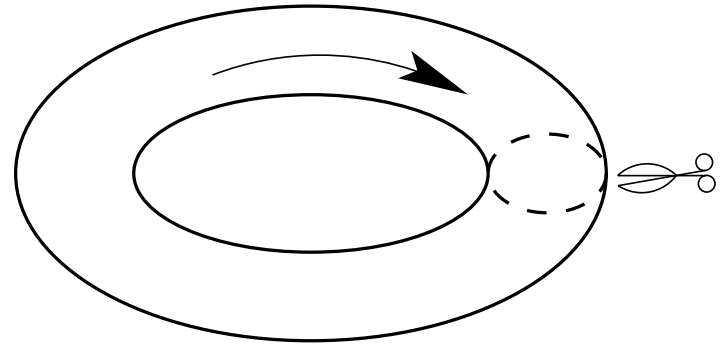

(a) Cutting

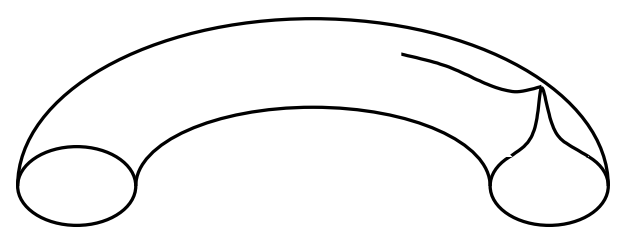

(c) Pinching \& stretching

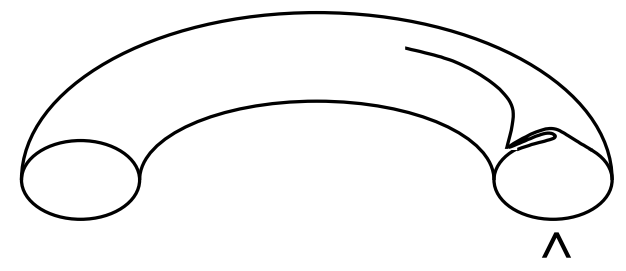

(e) Squeezing

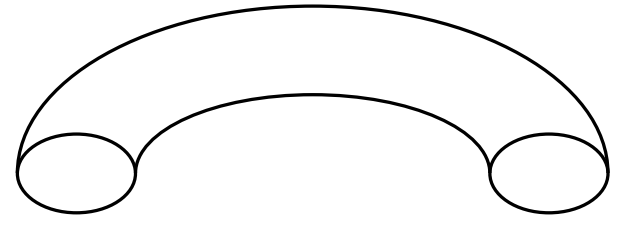

(b) Opening

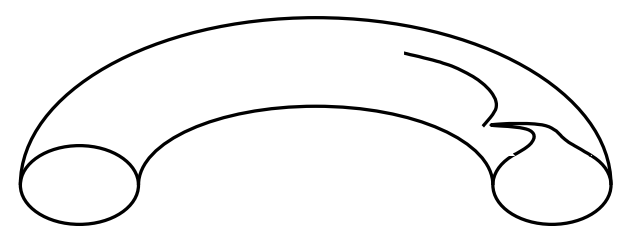

(d) Folding

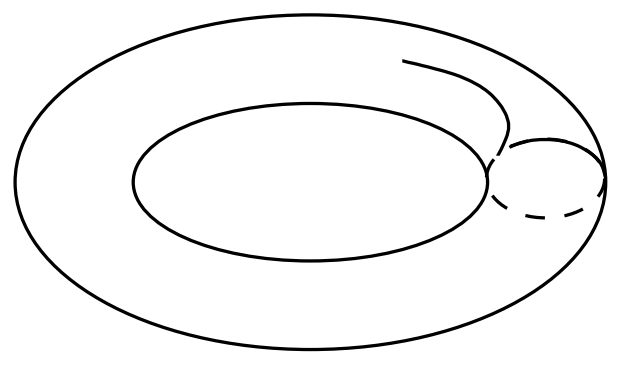

(f) Gluing 


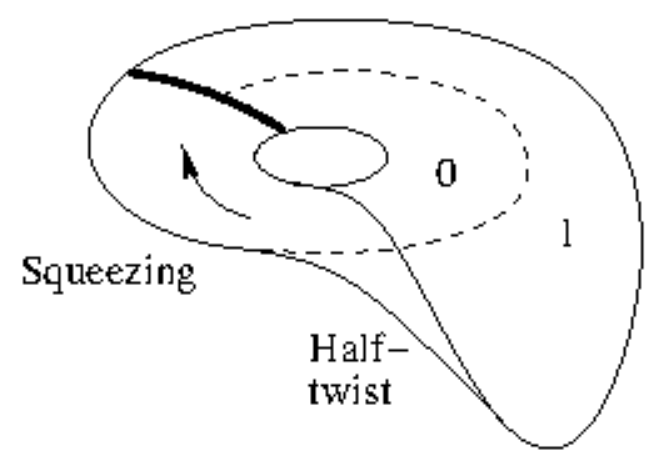

(a) Paper sheet model

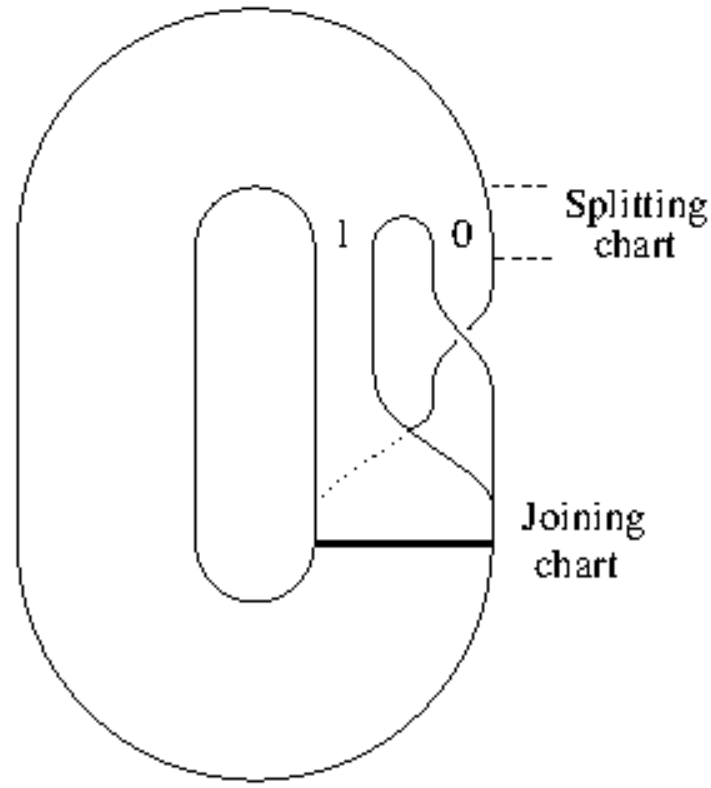

(b) Direct branched manifold

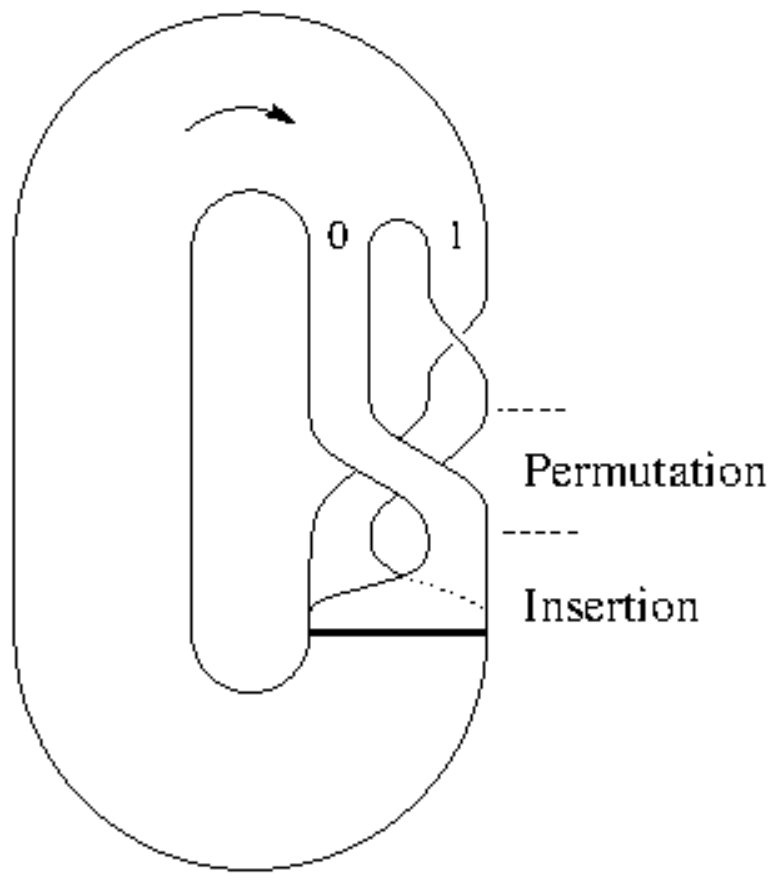

(c) Standard branched manifold 

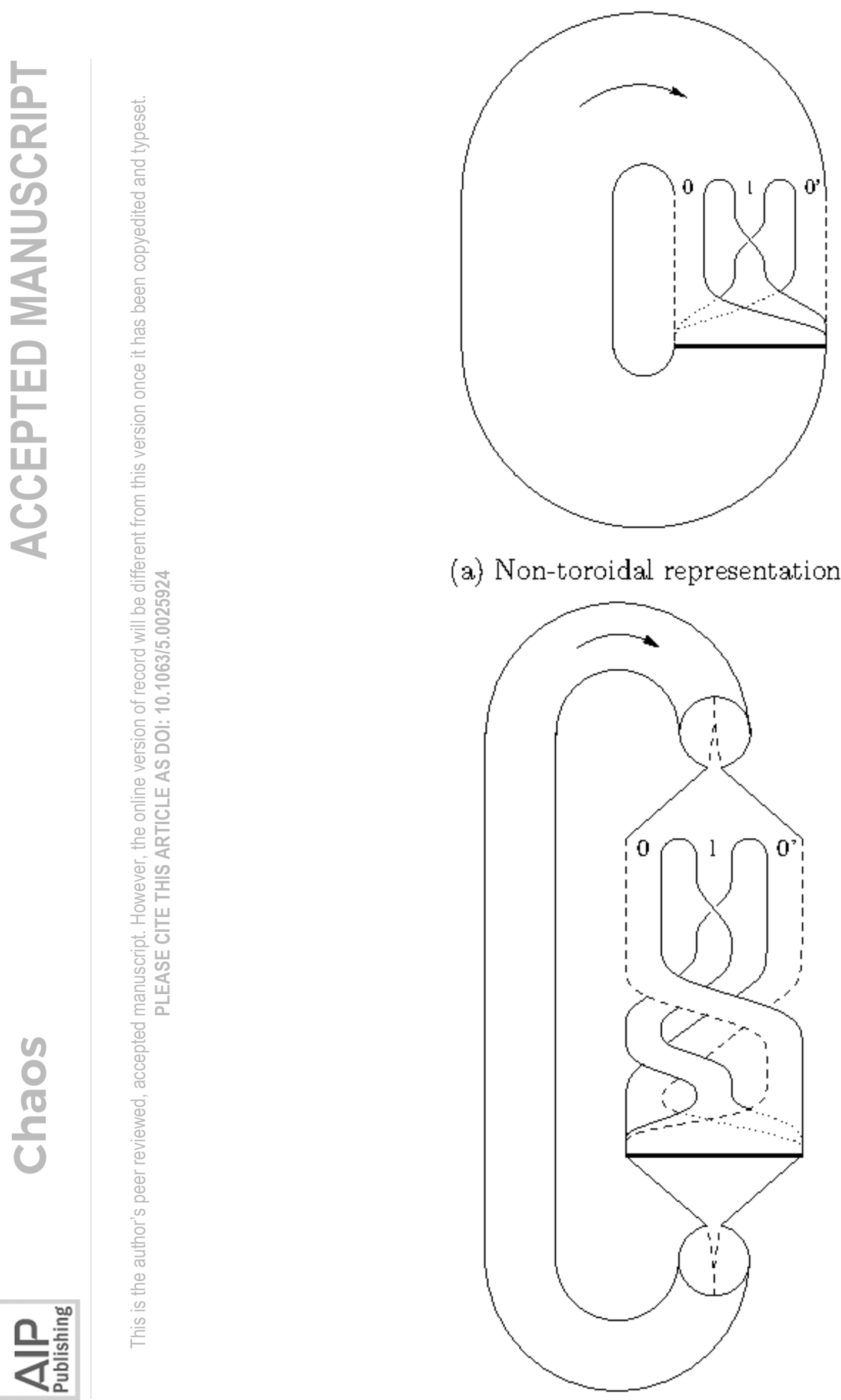

(a) Non-toroidal representation

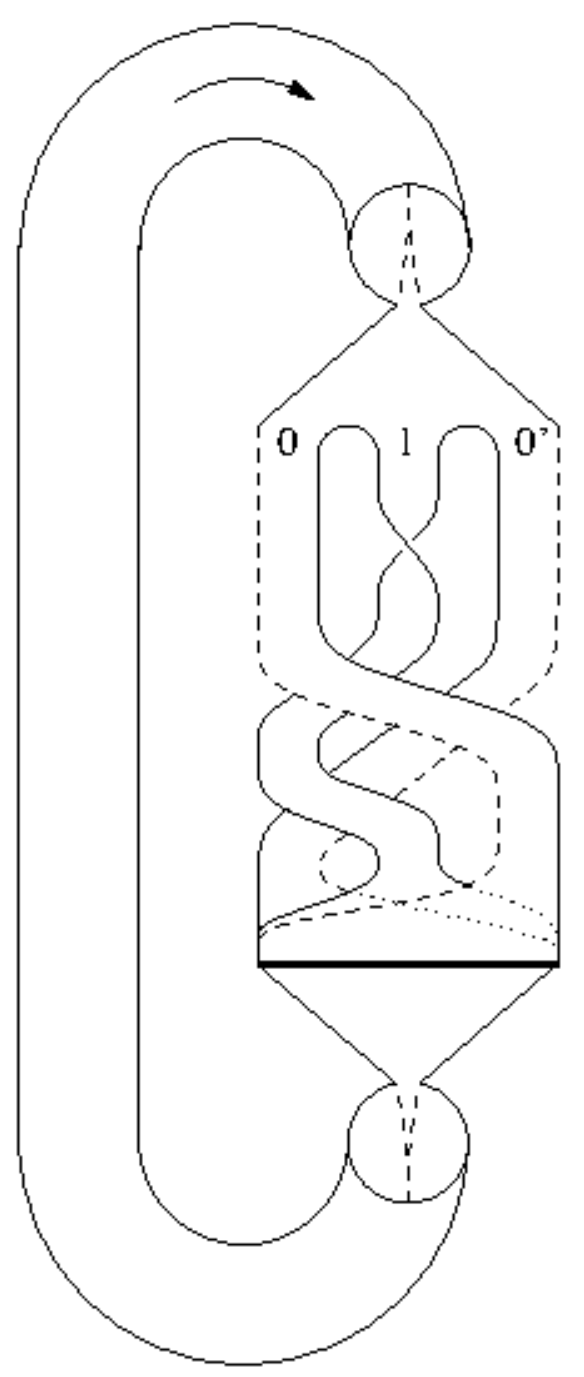

(b) Toroidal representation 

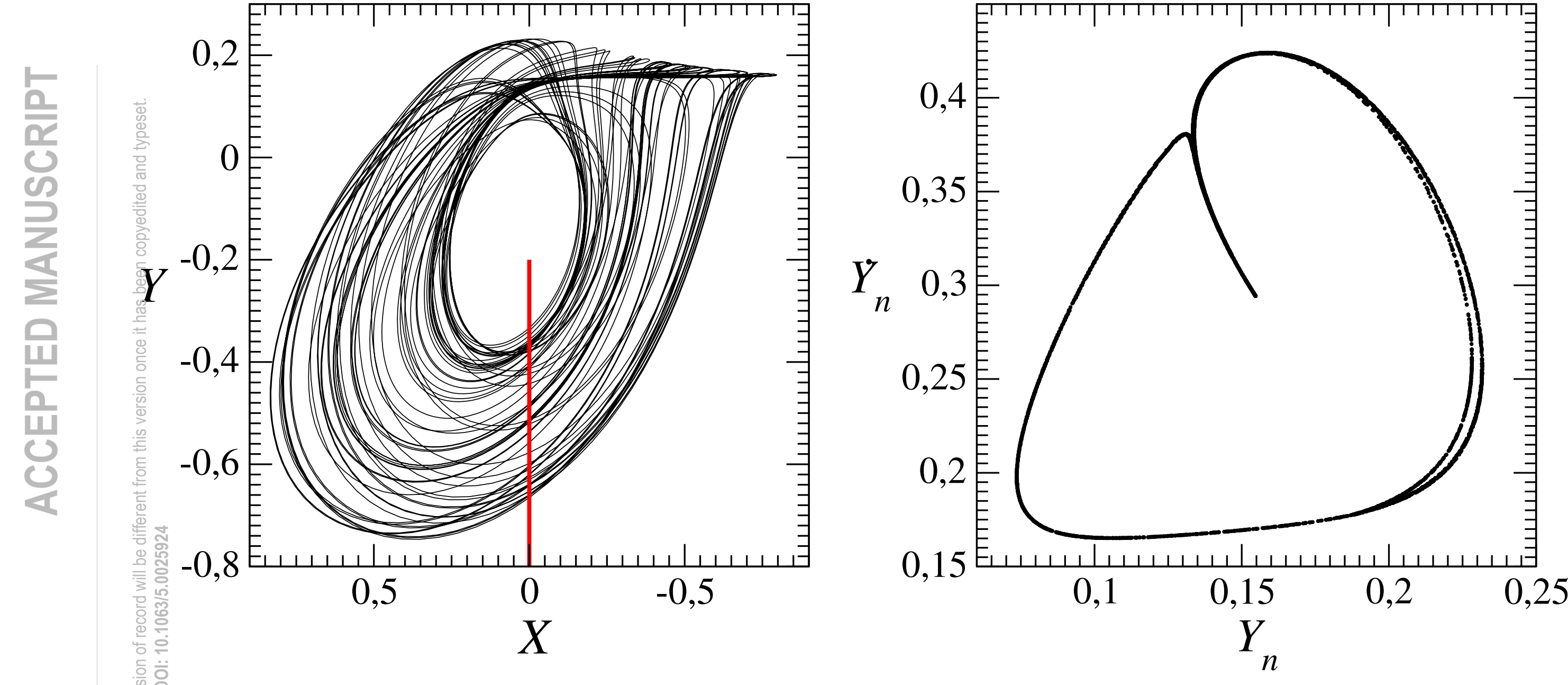


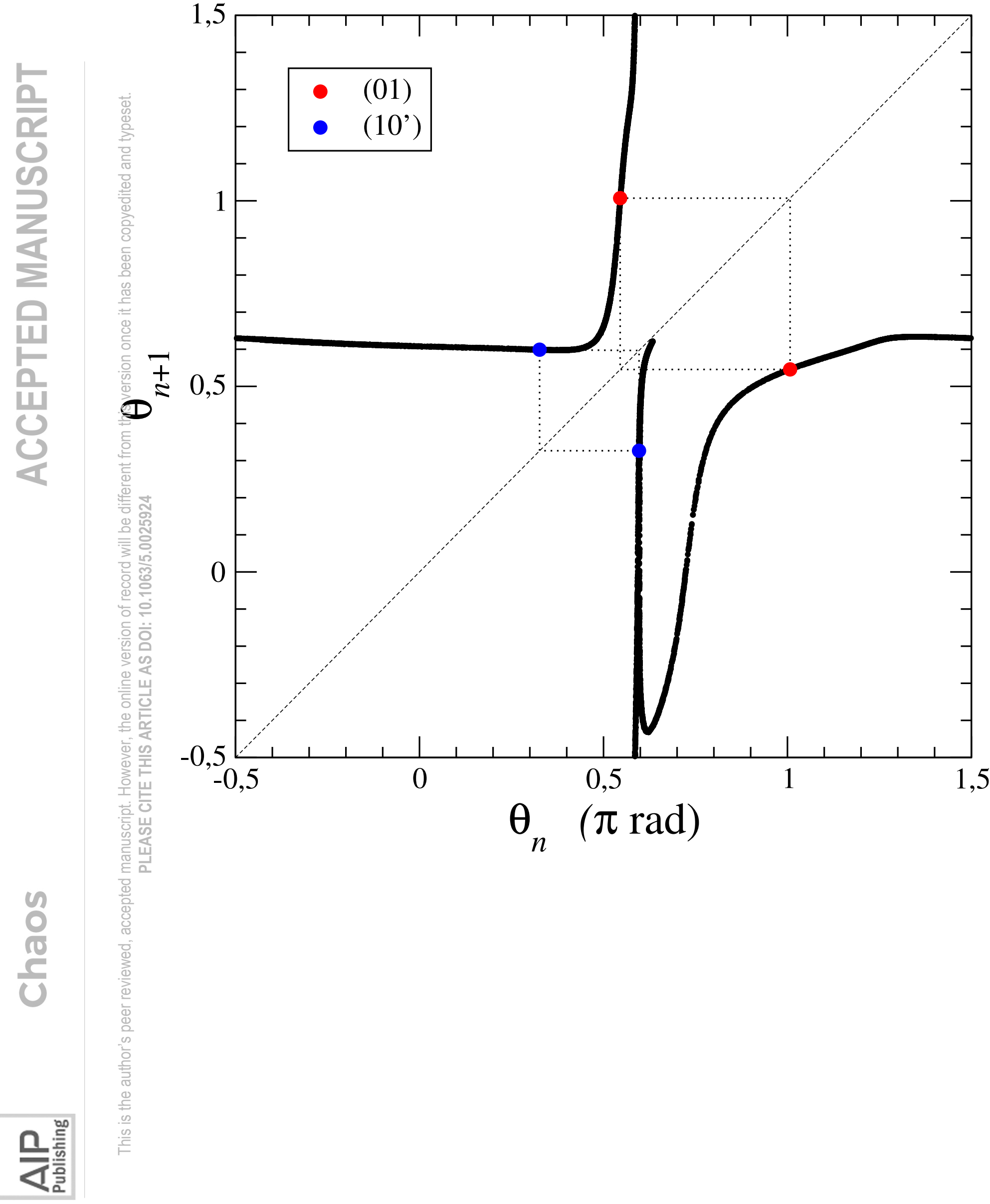




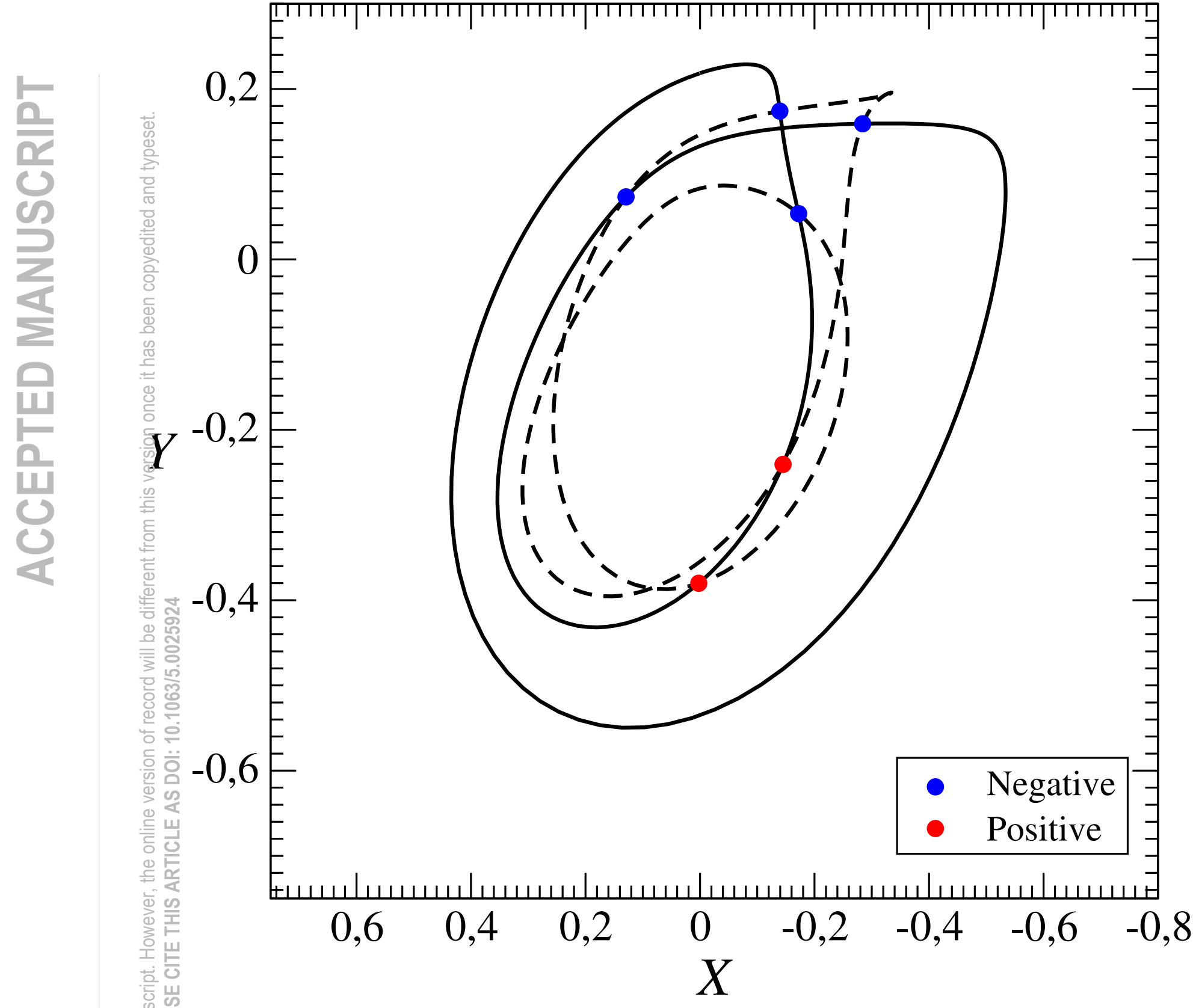




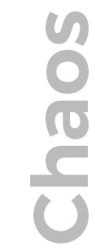

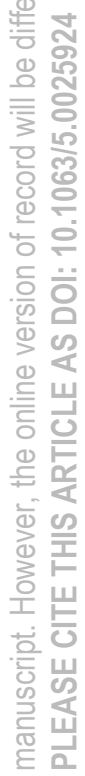




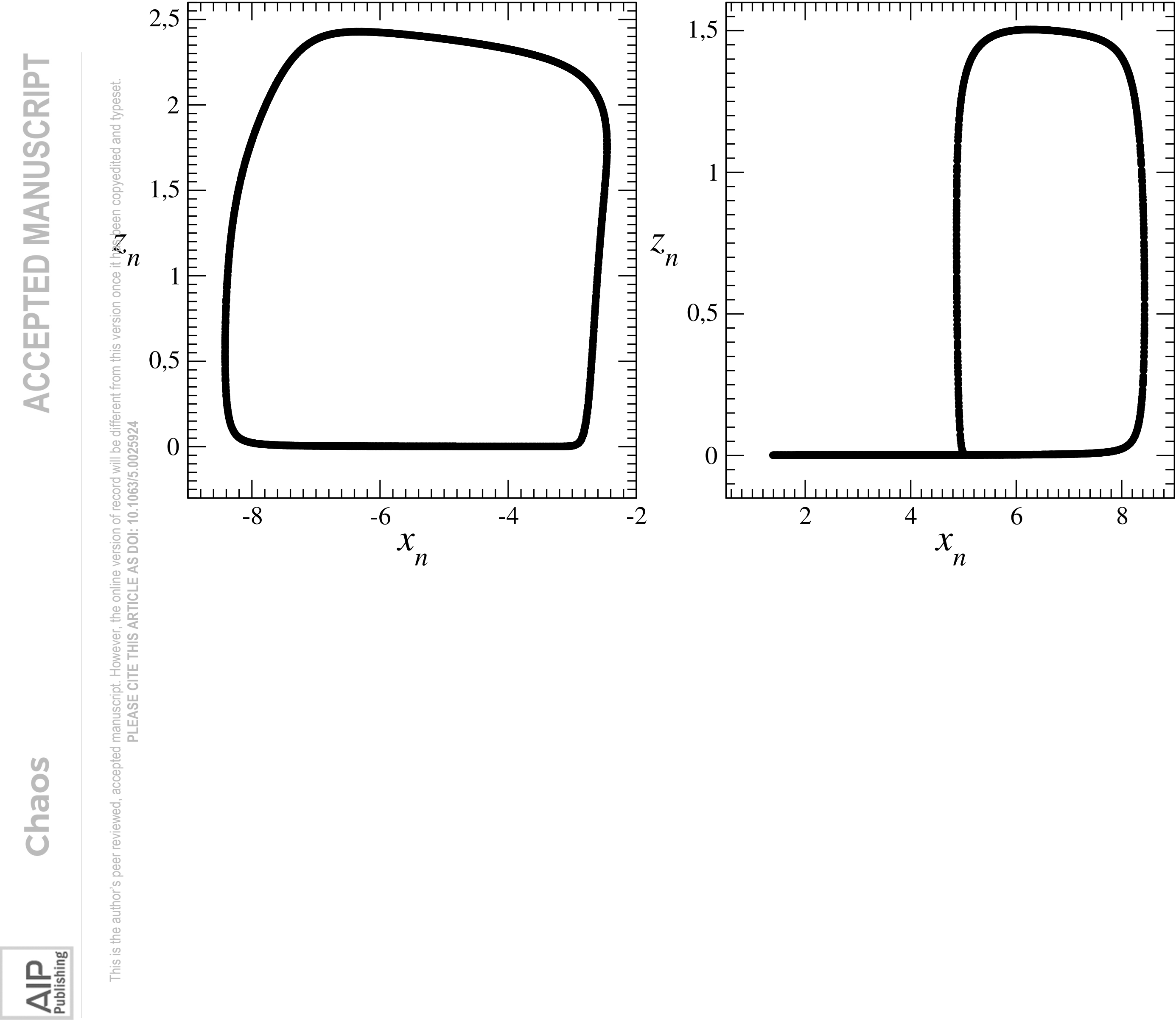




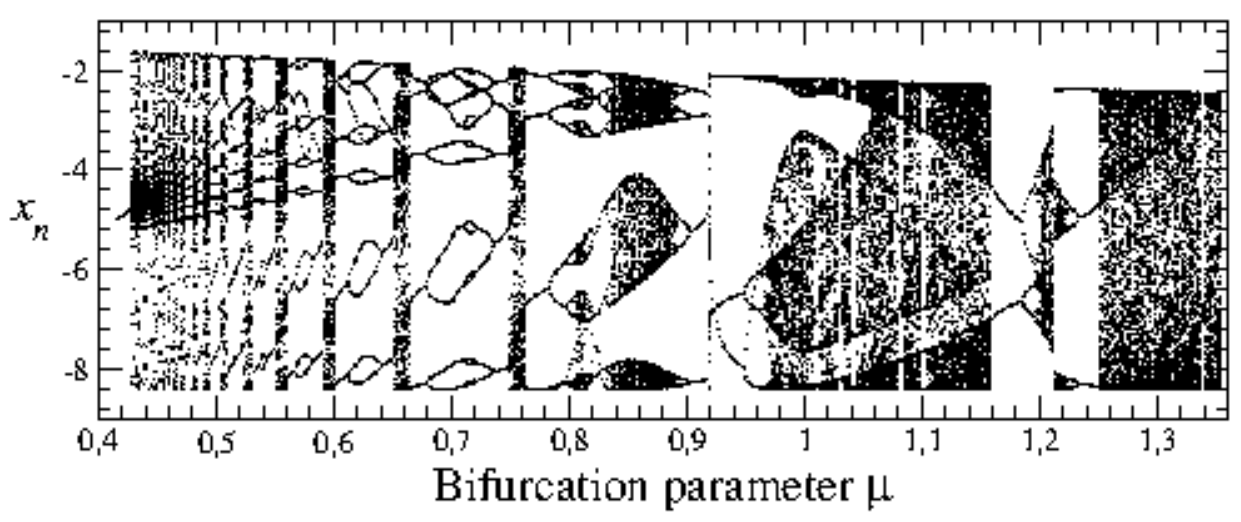

(a) Full diagram

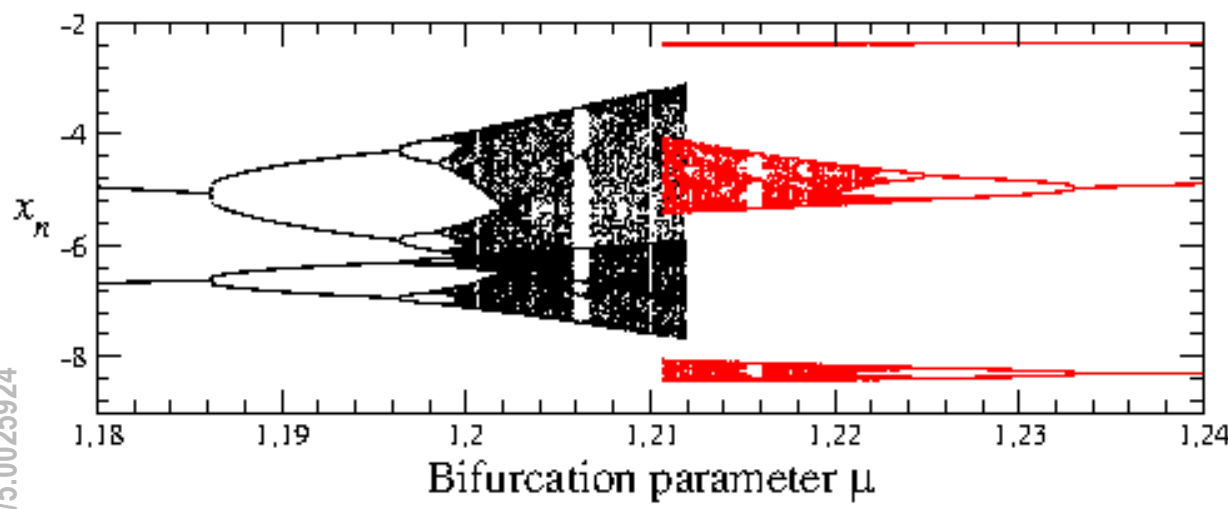

(b) Blow up 


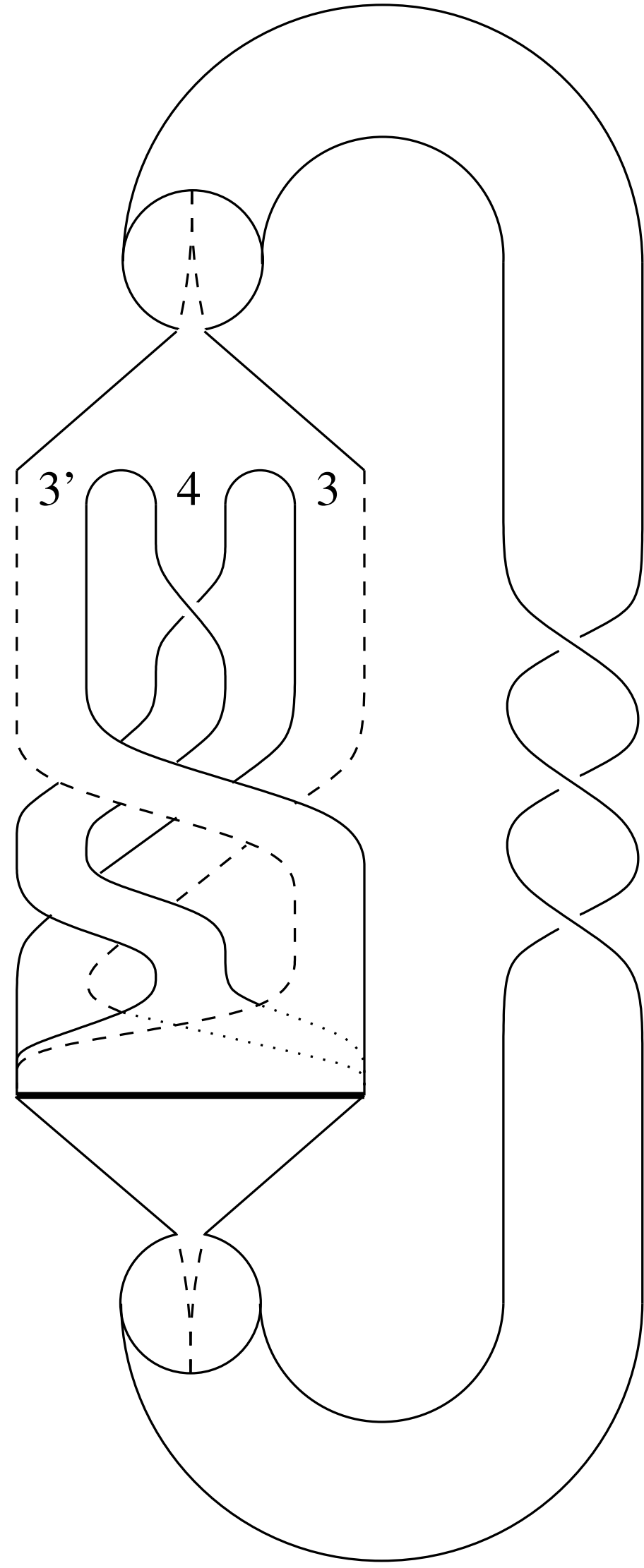




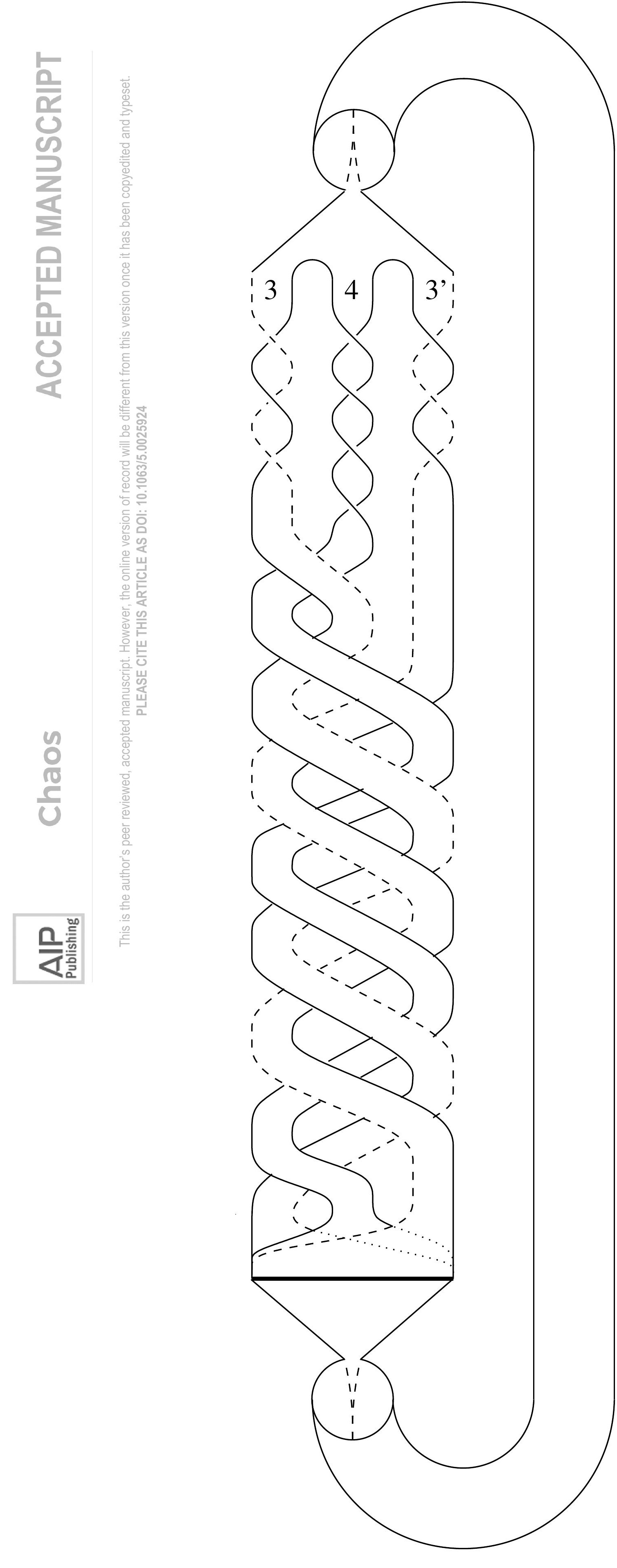




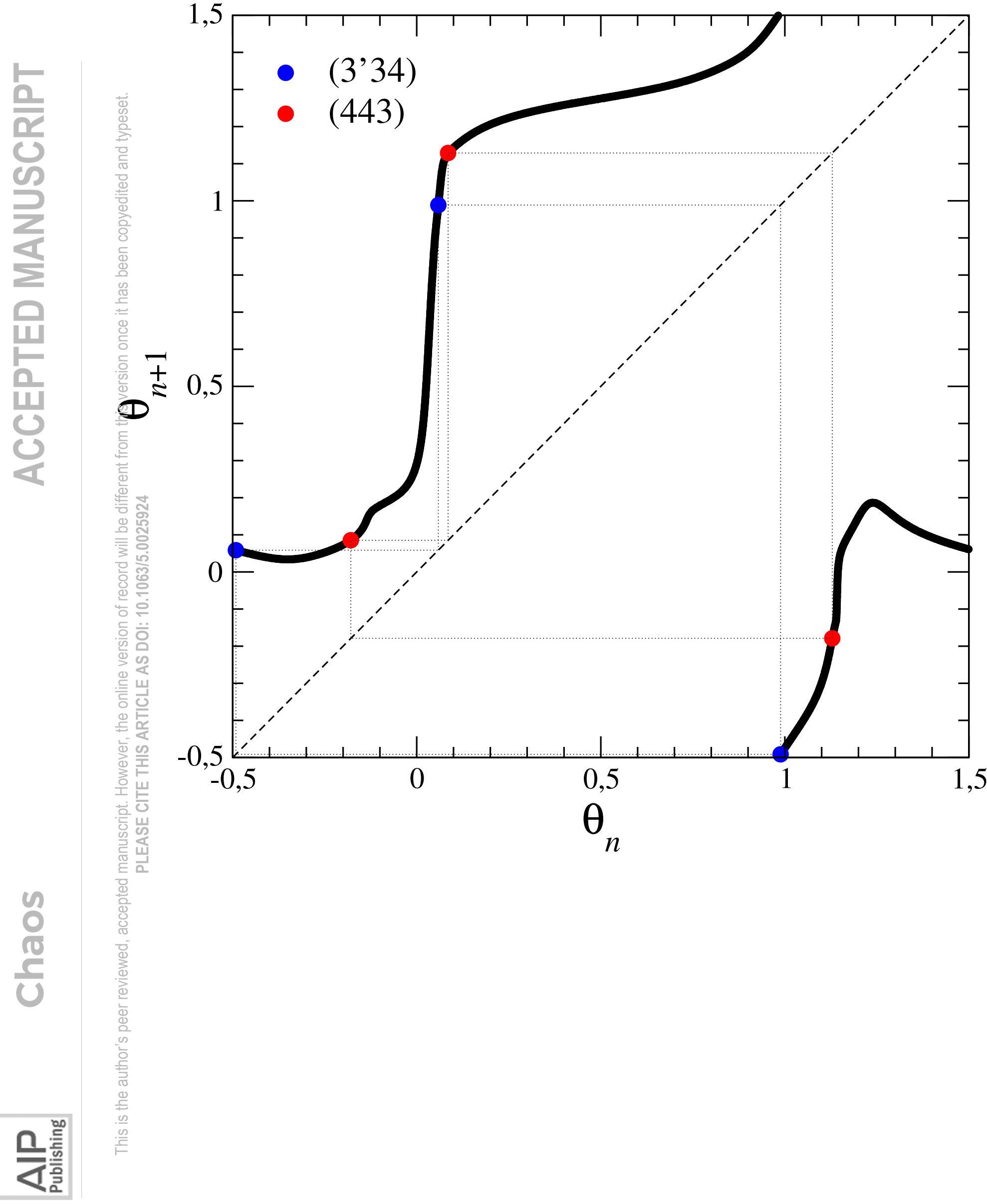




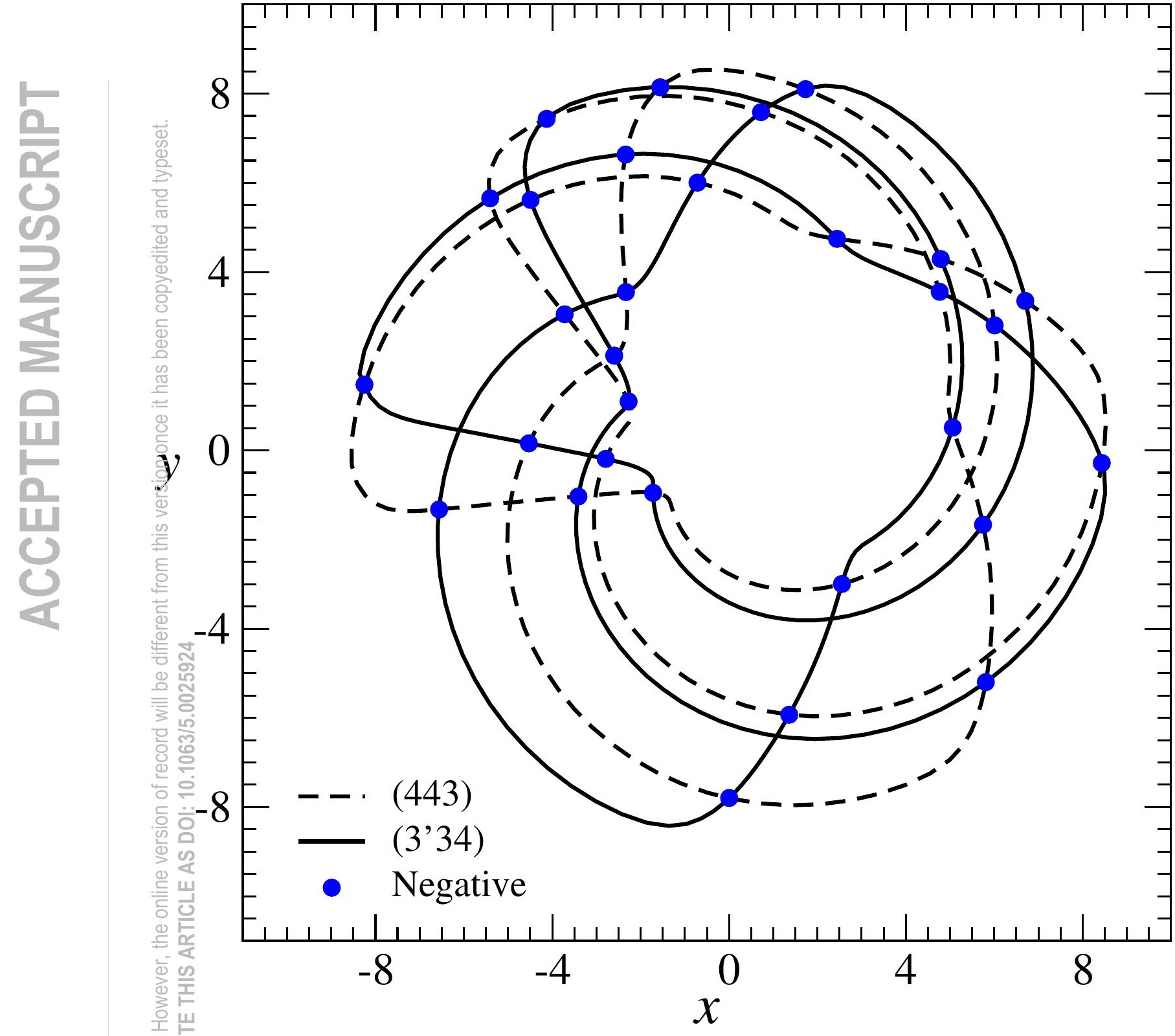

은 
